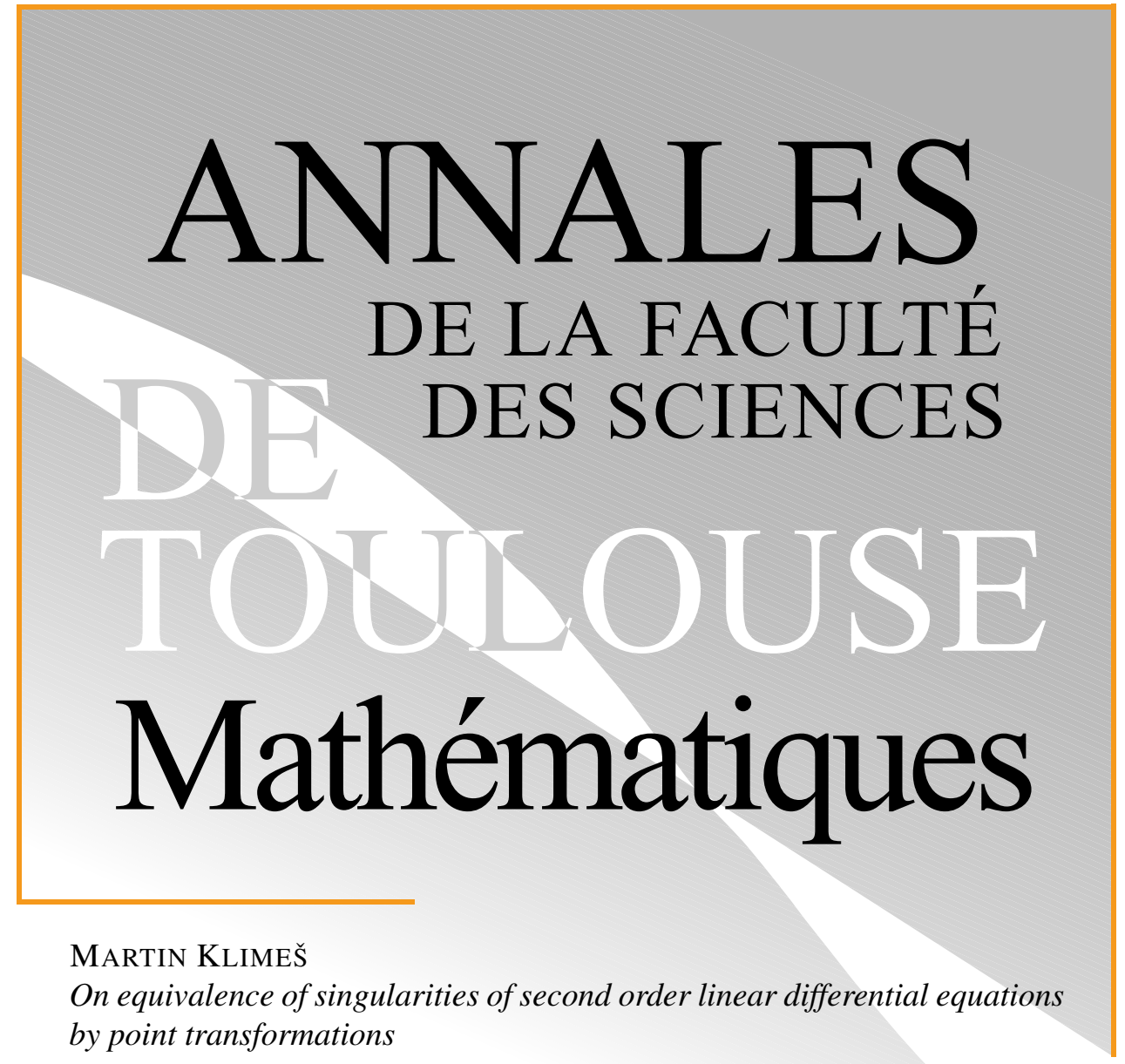

Tome XXX, no 3 (2021), p. 527-560.

https://doi.org/10.5802/afst.1684

(C) Université Paul Sabatier, Toulouse, 2021.

L'accès aux articles de la revue «Annales de la faculté des sciences de Toulouse Mathématiques » (http://afst.centre-mersenne.org/) implique l'accord avec les conditions générales d'utilisation (http://afst.centre-mersenne.org/legal/). Les articles sont publiés sous la license CC-BY 4.0.

(c) (i)

Publication membre du centre

Mersenne pour l'édition scientifique ouverte MERSENNE http://www.centre-mersenne.org/ 


\title{
On equivalence of singularities of second order linear differential equations by point transformations ${ }^{(*)}$
}

\author{
MARTIN KLIMEŠ (1)
}

\begin{abstract}
The article provides a local classification of singularities of meromorphic second order linear ordinary differential equations with respect to analytic/meromorphic linear point transformations, that is, transformations of both the unknown function and of the independent variable. In particular, it is shown that under a non-degeneracy condition two linear differential equations are analytically equivalent if and only if the associated companion systems are analytically equivalent as systems. Also the Lie algebras of analytic linear infinitesimal symmetries of the singularities are determined.
\end{abstract}

RÉsumé. - L'article propose une classification locale des singularités des équations différentielles linéaires du second ordre aux coefficients méromorphes par rapport aux transformations ponctuelles analytiques/méromorphes, c'est-à-dire, les transformations de la fonction inconnue aussi que de la variable indépendante. En particulier, il est montré que sous une condition de non-dégénérescence deux équations différentielles linéaires sont analytiquement équivalentes si et seulement si les systèmes compagnons associés sont analytiquement équivalents comme systèmes. Aussi les algèbres de Lie des symétries linéaires analytiques infinitésimales des singularités sont déterminées.

\section{Introduction}

Considering a second order linear differential equation (shortly LDE) on a complex domain $\Omega \subseteq \mathbb{C}$

$$
\frac{\mathrm{d}^{2} y}{\mathrm{~d} x^{2}}+a_{1}(x) \frac{\mathrm{d} y}{\mathrm{~d} x}+a_{0}(x) y=0, \quad(x, y) \in \Omega \times \mathbb{C},
$$

$\left.{ }^{*}\right)$ Reçu le 8 avril 2019, accepté le 10 septembre 2019.

Keywords: Linear ordinary differential equations, local analytic classification, normal forms, regular singularity, irregular singularity, Stokes phenomenon, analytic Lie symmetries.

(1) University of Zagreb, Faculty of Electrical Engineering and Computing, Unska 3, 10000 Zagreb, Croatia — martin.klimes@fer.hr

Article proposé par Vincent Guedj. 
with meromorphic coefficients $a_{1}(x), a_{0}(x)$, one can associate to it its companion linear differential system for $v={ }^{\mathrm{t}}\left(y, \frac{\mathrm{d} y}{\mathrm{~d} x}\right)$

$$
\frac{\mathrm{d} v}{\mathrm{~d} x}=\left(\begin{array}{cc}
0 & 1 \\
-a_{0} & -a_{1}
\end{array}\right) v, \quad(x, v) \in \Omega \times \mathbb{C}^{2} .
$$

And vice versa every $2 \times 2$ linear differential system with meromorphic coefficients can be meromorphically transformed to the form of a companion system (1.2) by virtue of the so called "cyclic vector lemma" [21, §2.1]. From the point of study of the solutions there is not much distinction between meromorphic second order LDEs (1.1) and $2 \times 2$ meromorphic differential systems (1.2). Correspondingly, the two are also interchangeable from the point of view of differential Galois theory. However, there is an important difference between them when it comes to the underlying space and to its transformation group, and consequently also when it comes to their Lie symmetry groups.

A meromorphic linear differential system

$$
\frac{\mathrm{d} v}{\mathrm{~d} x}=A(x) v, \quad(x, v) \in \Omega \times \mathbb{C}^{2},
$$

can be seen as a meromorphic connection on a (trivial) rank 2 vector bundle over $\Omega$. As such, a natural kind of transformations of the system are the gauge-coordinate transformations

$$
\widetilde{x}=\phi(x), \quad \widetilde{v}=T(x) v,
$$

with $\phi: \Omega \rightarrow \Omega$ an analytic diffeomorphism and $T(x)$ a matrix-valued function that is either analytic and analytically invertible, or meromorphic and meromorphically invertible. The corresponding systems for $v$ and $\widetilde{v}$ are then called analytically, resp. meromorphically, equivalent. In the literature one often considers the above transformations with $\phi=\mathrm{id}_{\Omega}$, but here we opt for the more general form (1.4).

On the other hand, a second order LDE (1.1) is living as a connection on the 1-jet bundle $\mathcal{J}^{1}(\Omega)$ (or more generally on a codimension 1 subbundle of the 2 -jet bundle $\mathcal{J}^{2}(\Omega)$, cf. $\left.[9, \S 19 \mathrm{E}]\right)$. A natural kind of transformations are linear point transformations, that is the jet prolongations of the transformations

$$
\widetilde{x}=\phi(x), \quad \widetilde{y}=t(x) y,
$$

with $\phi: \Omega \rightarrow \Omega$ analytic diffeomorphism and $t(x)$ either a non-vanishing analytic, or a non-zero meromorphic, function. Such transformations were considered already by Kummer [11], and it is known that general point transformations preserving the class of second (or higher) order LDEs are locally of the this form [23]. The corresponding equations for $y$ and $\widetilde{y}$ are then called analytically, resp. meromorphically, equivalent. 
For higher order differential equations the group of transformations (1.5) is very restrictive, therefore more general transformations are often considered such as "Weyl transformations" (generalized linear contact transformations) $[9,25]$

$$
\widetilde{x}=\phi(x), \quad \widetilde{y}=t_{0}(x) y+\ldots+t_{n-1}(x)\left(\frac{\partial}{\partial x}\right)^{n-1} y,
$$

where $n$ is the order of the LDE. Nevertheless the case $n=2$, that we consider here, is special in the sense that the dimension of the solutions space is the same as the dimension of the ambient $(x, y)$-space, and locally the linear point transformations (1.5) are relatively rich enough to provide, at least in a generic situation, a satisfactory theory.

In this paper, we are interested in local analytic classification of meromorphic second order LDEs (1.1) with respect to linear point transformations (1.5) near a fixed point in the $x$-space placed at the origin, i.e. $\Omega=(\mathbb{C}, 0)$. If both coefficients $a_{0}(x), a_{1}(x)$ of $(1.1)$ are analytic at $x=0$, then it is well known [12] that the equation is locally analytically equivalent to

$$
\frac{\mathrm{d}^{2} y}{\mathrm{~d} x^{2}}=0 \text {. }
$$

We will therefore investigate only singular points, where at least one of $a_{0}(x), a_{1}(x)$ has a pole.

While the local analytic/meromorphic theory of meromorphic linear differential systems with respect gauge transformations $\widetilde{v}=T(x) v$, fixing the coordinate $x$, is well established by now, e.g. [1, 2, 3, 9, 21], and it is easily generalized to all transformation (1.4), there appears to be very little written on the subject of local analytic/meromorphic classification of singular second-order LDEs with respect to linear point transformations. We will describe the local analytic/meromorphic moduli space of LDEs (1.1) at singularities of regular or non-resonant irregular type, as well as those that become non-resonant irregular in the ramified coordinate $x^{\frac{1}{2}}$, (Sections 1.2/1.3), and describe their Lie groups of linear infinitesimal symmetries (Section 1.4).

\subsection{Singularities of linear differential equations}

Suppose that at least one coefficient $a_{i}(x), i=0,1$, of (1.1) has a pole at the origin, and denote $m_{i} \in \mathbb{Z}$ the respective orders of the poles. Let

$$
\nu=\max \left\{m_{1}-1,\left\lceil\frac{m_{0}}{2}\right\rceil-1\right\} \geqslant 0
$$


we will call it the Poincaré rank of the LDE. Denoting

$$
\delta_{\nu}:=x^{\nu+1} \frac{\partial}{\partial x},
$$

and rewriting the equation (1.1) as

$$
\delta_{\nu}^{2} y-p(x) \delta_{\nu} y-q(x) y=0,
$$

where $p(x)=-x^{\nu+1} a_{1}(x)+(\nu+1) x^{\nu}$ and $q(x)=-x^{2 \nu+2} a_{0}(x)$, then the Poincaré rank $\nu \geqslant 0$ is the smallest integer for which $p(x), q(x)$ are analytic at 0 .

The companion system for (1.6) is the system for $v={ }^{\mathrm{t}}\left(y, \delta_{\nu} y\right)$

$$
\delta_{\nu} v=\left(\begin{array}{cc}
0 & 1 \\
q(x) & p(x)
\end{array}\right) v .
$$

The equation (1.6) can be rewritten as

$$
\left(\delta_{\nu}-\frac{p(x)}{2}\right)^{2} y=\frac{\Delta(x)}{4} y, \quad \text { where } \Delta:=p^{2}+4 q-2 \delta_{\nu} p .
$$

An equation (1.6) with $p(x)=0$ will be called trace-free.

DEFINITION.

(1) If $\nu=0$, then the singularity is called regular or Fuchsian (by a theorem of Fuchs the notions of regular singularity and Fuchsian singularity coincide for LDEs [9, Theorem 19.20]). A Fuchsian singularity is strictly non-resonant if the roots of $\lambda^{2}-p(0) \lambda-q(0)=0$ do not differ by an integer, ${ }^{(1)}$ i.e. if $\sqrt{\Delta(0)} \notin \mathbb{Z}$, otherwise it is called resonant.

(2) If $\nu>0$, then the singularity is called irregular. An irregular singularity is non-resonant if the roots of $\lambda^{2}-p(0) \lambda-q(0)=0$ are distinct; i.e. if $\Delta(0) \neq 0$.

It will be called non-degenerate if

- either $\Delta(0) \neq 0$ (non-resonant),

- or $\Delta(0)=0$ and $p(0)=0$, in which case $\frac{\mathrm{d} \Delta}{\mathrm{d} x}(0) \neq 0$ (otherwise the Poincaré rank would be lower than $\nu)$.

This is equivalent to say that in the coordinate $s=x^{\frac{1}{2}}$ the LDE has a non-resonant irregular singularity.

We shall prove the following result relating the formal and the analytic equivalence of singular LDEs with that of their companion systems (1.7).

(1) This condition is slightly stronger than the usual non-resonance condition that demands that the roots do not differ by a non-zero integer [9, Definition 16.12]. 
THEOREM 1.1. - Two LDEs (1.6) with either a regular singularity or a non-degenerate irregular singularity at the origin are analytically, resp. formally, equivalent if and only if the companion systems (1.7) are analytically, resp. formally, equivalent.

Let us stress that we consider equivalence by linear point transformations (1.5) for equations, and by gauge-coordinate transformations (1.4) for systems. By formal equivalence it is meant that the diffeomorphism $\phi(x)$ and the function $t(x)$ in (1.5), resp. $T(x)$ in (1.4), are formal power series of $x$.

On the other hand, for degenerate irregular singularities it is possible to have analytically inequivalent LDEs with analytically equivalent companion systems (Example 2.1).

In the following section we describe the moduli space of analytic equivalence of regular and non-degenerate irregular singularities of LDEs. In the light of Theorem 1.1, these results are parallel to the classical theory of singularities of linear differential systems and are a direct reformulation of it.

\subsection{Formal and analytic classification}

It is easy to verify that if $y_{1}(x), y_{2}(x)$ are two linearly independent solutions to (1.6) then one can express $\Delta(x)(1.8)$ as

$$
\Delta(x)=-2 \mathcal{S}_{\nu}\left(\frac{y_{1}}{y_{2}}\right)(x), \quad \text { where } \mathcal{S}_{\nu}(f)=\delta_{\nu}\left(\frac{\delta_{\nu}^{2} f}{\delta_{\nu} f}\right)-\frac{1}{2}\left(\frac{\delta_{\nu}^{2} f}{\delta_{\nu} f}\right)^{2}
$$

is the "Schwarzian derivative" associated to $\delta_{\nu}$. The point transformation (1.5) relate two such LDEs by

$$
\begin{aligned}
p & =\psi \cdot \widetilde{p} \circ \phi+\frac{\delta_{\nu} \psi}{\psi}-2 \frac{\delta_{\nu} t}{t}, \\
q & =\psi^{2} \cdot \widetilde{q} \circ \phi+\left[\psi \cdot \widetilde{p} \circ \phi+\frac{\delta_{\nu} \psi}{\psi}\right] \frac{\delta_{\nu} t}{t}-\frac{\delta_{\nu}^{2} t}{t}, \\
\Delta & =\psi^{2} \cdot \widetilde{\Delta} \circ \phi-2 \delta_{\nu}\left(\frac{\delta_{\nu} \psi}{\psi}\right)+\left(\frac{\delta_{\nu} \psi}{\psi}\right)^{2},
\end{aligned}
$$

where $\psi(x)=\frac{\delta_{\nu} \phi}{\phi^{\nu+1}}$, i.e. $\delta_{\nu}=\psi(x) \widetilde{\delta}_{\nu}$. In fact, the formula (1.11) is just the transformation rule for the above "Schwarzian derivative"

$$
\mathcal{S}_{\nu}(f \circ \phi)=\psi^{2} \cdot \mathcal{S}_{\nu}(f) \circ \phi+\delta_{\nu}\left(\frac{\delta_{\nu} \psi}{\psi}\right)-\frac{1}{2}\left(\frac{\delta_{\nu} \psi}{\psi}\right)^{2} .
$$


In this notation, the operator $\mathcal{S}_{-1}$ is the usual Schwarzian derivative for which the transformation rule reads $\mathcal{S}_{-1}(f \circ \phi)=\left(\frac{\mathrm{d} \phi}{\mathrm{d} x}\right)^{2} \cdot \mathcal{S}_{-1}(f) \circ \phi+\mathcal{S}_{-1}(\phi)$, and

$$
\Delta(x)=-2 x^{2 \nu+2} \mathcal{S}_{-1}\left(\frac{y_{1}}{y_{2}}\right)-\left(\nu^{2}-1\right) x^{2 \nu} .
$$

Remark. - The point transformations preserving the space of trace-free LDEs $\delta_{\nu}^{2} y=\frac{\Delta(x)}{4} y$ are of the form $x \mapsto \phi(x), y \mapsto c \psi(x)^{\frac{1}{2}} y$ with $\psi(x)=$ $\frac{\delta_{\nu} \phi}{\phi^{\nu}+1}$, i.e. up to the multiplication by a constant $c \in \mathbb{C} \backslash\{0\}$ they act as transformations of the $\left(-\frac{1}{2}\right)$-differential $y(x) \delta_{\nu}^{\frac{1}{2}}$, as was observed in [8].

Notation. - While we denote $\delta_{\nu}^{2}=\delta_{\nu} \delta_{\nu}, \delta_{\nu}^{3}=\delta_{\nu} \delta_{\nu} \delta_{\nu}$ etc. the higher order differential operators defined by composition of $\delta_{\nu}$, at the same time we also write $\delta_{\nu}^{-2}=\frac{(\mathrm{d} x)^{2}}{x^{2 \nu+2}}$, and $\delta_{\nu}^{\frac{1}{2}}=x^{\frac{\nu+1}{2}}(\mathrm{~d} x)^{-\frac{1}{2}}$ for formal powers of the differential form $\delta_{\nu}^{-1}=\frac{\mathrm{d} x}{x^{\nu+1}}$. This double convention should not cause any confusion.

From (1.9) and (1.11) one can see that

$$
p(x)=\psi(x) \cdot \widetilde{p}(\phi(x))+O\left(x^{\nu+1}\right), \quad \Delta(x)=\psi(x)^{2} \cdot \widetilde{\Delta}(\phi(x))+O\left(x^{2 \nu+1}\right) .
$$

The natural objects to consider as invariants are the meromorphic form $J_{0}^{\nu} p(x) \cdot \delta_{\nu}^{-1}$ and the meromorphic quadratic differential $J_{0}^{2 \nu} \Delta(x) \cdot \delta_{\nu}^{-2}$, where $J_{0}^{k}$ denotes the $k$-jet of a germ at $x=0$, on which the point transformations act as the usual transformation rule for differentials.

If $\Delta(0) \neq 0$, let

$$
\mu^{2}=\left(\operatorname{Res}_{x=0} \sqrt{\Delta(x)} \delta_{\nu}^{-1}\right)^{2},
$$

be the square residue of the quadratic differential $\Delta(x) \delta_{\nu}^{-2}$. The following proposition is well known in literature, see e.g. [24, Theorems $6.1,6.3,6.4]$.

Theorem 1.2 (Local normal form of the quadratic differential $\Delta(x) \delta_{\nu}^{-2}$ ).

(1) If $\nu=0$ and $\mu^{2}=\Delta(0) \neq 0$ then there exists an analytic transformation $x \mapsto \phi(x)$ such that in the new variable

$$
\Delta(x) \delta_{0}^{-2}=\mu^{2} \delta_{0}^{-2} .
$$

(2) If $\nu>0$ then there exists an analytic transformation $x \mapsto \phi(x)$ such that in the new variable

$$
\Delta(x) \delta_{\nu}^{-2}=\left(1+\mu x^{\nu}\right)^{2} \delta_{\nu}^{-2},
$$

where $\mu \in \mathbb{C}$ is a root of the square residue $\mu^{2}$, determined up to the \pm sign. 
(3) If $\Delta(0)=0, \frac{\mathrm{d} \Delta}{\mathrm{d} x}(0) \neq 0$, then there exists an analytic transformation $x \mapsto \phi(x)$ such that in the new variable

$$
\Delta(x) \delta_{\nu}^{-2}=x \delta_{\nu}^{-2} .
$$

In the situation we consider (regular or non-degenerate irregular singularities) it turns out that the simultaneous equivalence class of the pair of jets of forms $J_{0}^{\nu} p(x) \cdot \delta_{\nu}^{-1}$ and $J_{0}^{2 \nu} \Delta(x) \cdot \delta_{\nu}^{-2}$ is determined by the pair $J_{0}^{\nu} p(x) \cdot \delta_{\nu}^{-1}$ and $J_{0}^{\nu} \Delta(x) \cdot \delta_{\nu}^{-2}$.

Definition (Formal invariants). - Let $J_{0}^{k} f(x)$ denote the $k$-jet of a germ $f(x)$ at $x=0$.

We define the formal invariant of the $L D E$ (1.6) as the simultaneous equivalence class of the pair of meromorphic forms

$$
J_{0}^{\nu} p(x) \cdot \delta_{\nu}^{-1}, \quad J_{0}^{\nu} \Delta(x) \cdot \delta_{\nu}^{-2},
$$

with respect to the action of analytic diffeomorphisms $x \mapsto \phi(x)$ and jet restriction.

- If $\nu=0$, then the formal invariant is given by the pair $p(0), \Delta(0)=$ $\mu^{2}$, and will be identified with the pair of roots $\left\{\lambda_{1}, \lambda_{2}\right\}$ of $\lambda^{2}-$ $p(0) \lambda-q(0)=0$.

- If $\nu>0$ and the irregular singularity is non-resonant, $\Delta(0) \neq 0$, let

$$
\lambda_{j}(x)=\lambda_{j}^{(0)}+\cdots+x^{\nu} \lambda_{j}^{(\nu)}, \quad j=1,2,
$$

be the $\nu$-jets of the roots of the characteristic polynomial $\lambda^{2}-p(x) \lambda-$ $q(x)$. Then $J_{0}^{\nu} p(x)=\lambda_{1}(x)+\lambda_{2}(x), J_{0}^{\nu} \sqrt{\Delta}(x)=\lambda_{2}(x)-\lambda_{1}(x)$, and the formal invariant can be identified with the equivalence class of the pair

$$
\left\{\lambda_{1}(x) \delta_{\nu}^{-1}, \lambda_{2}(x) \delta_{\nu}^{-1}\right\}
$$

with respect to the action of analytic diffeomorphisms $x \mapsto \phi(x)$ and jet restriction. Moreover, one may always assume that $\Delta(x) \delta_{\nu}^{-2}=$ $\left(1+\mu x^{\nu}\right)^{2} \delta_{\nu}^{-2}$ is in the normal form of Proposition 1.2 and

$$
\lambda_{2}(x)-\lambda_{1}(x)=1+\mu x^{\nu} .
$$

Such pair $\left\{\lambda_{1}(x) \delta_{\nu}^{-1}, \lambda_{2}(x) \delta_{\nu}^{-1}\right\}$, called in canonical form, is uniquely determined up to the action of the rotations $x \mapsto e^{\frac{l \pi i}{\nu}} x, l \in \mathbb{Z}_{2 \nu}$. In particular, if the equation is trace-free, then $\lambda_{1}(x)+\lambda_{2}(x)=0$, and the equivalence class of formal invariants is completely determined by $\nu$ and $\mu^{2}$.

- If $\nu>0, \Delta(0)=0$ and the resonant irregular singularity is nondegenerate, $\frac{\mathrm{d} \Delta}{\mathrm{d} x}(0) \neq 0$, then one can assume that $\Delta(x) \delta_{\nu}^{-2}=x \delta_{\nu}^{-2}$, in which case $J_{0}^{\nu} p(x) \cdot \delta_{\nu}^{-1}$ is uniquely determined up to the action of the rotations $x \mapsto e^{\frac{2 l \pi i}{2 \nu-1}} x, l \in \mathbb{Z}_{2 \nu-1}$. 
Definition. - A linear differential equation (1.6) is called reducible if it can be written as

$$
\left(\delta_{\nu}-\alpha_{2}(x)\right)\left(\delta_{\nu}-\alpha_{1}(x)\right) y=0,
$$

with $\alpha_{1}(x), \alpha_{2}(x)$ analytic, $\alpha_{j}(x)=\sum_{k=0}^{+\infty} \alpha_{j}^{(k)} x^{k}$.

\subsubsection{Regular singularities}

Definition (Projective monodromy). - Let $y_{1}(x), y_{2}(x)$ be two linearly independent solutions near a point $x_{0} \in U^{*}$ of some pointed neighborhood $U^{*}$ of the origin, and let $f(x):=\frac{y_{2}(x)}{y_{1}(x)}$. For a loop $\gamma \in \pi_{1}\left(U^{*}, x_{0}\right)$, the analytic continuation of $f(x)$ along $\gamma$ acts on $f(x)$ as

$$
f(\gamma \cdot x)=\rho_{\gamma}(f(x)), \quad \text { for some } \rho_{\gamma} \in \mathrm{PGL}_{2}(\mathbb{C}) .
$$

The map $\rho: \gamma \mapsto \rho_{\gamma}$ is the projective monodromy representation

$$
\rho: \pi_{1}\left(U^{*}, x_{0}\right) \rightarrow \mathrm{PGL}_{2}(\mathbb{C})
$$

of the LDE. This representation, which is the projectivization of the monodromy representation of the companion system, is well-defined up to conjugacy in $\mathrm{PGL}_{2}(\mathbb{C})$.

Let $\gamma_{0}$ be a positively oriented simple loop generating $\pi_{1}\left(U^{*}, x_{0}\right)$. Then $\rho_{\gamma_{0}}$ is conjugated to either

(i) $f \mapsto c f$, for some $c \in \mathbb{C}^{*}$, or

(ii) $f \mapsto f+1$.

In the case (i) we call the projective monodromy diagonalizable, and in the case (ii) non-diagonalizable.

LEMMA 1.3 .

(1) Strongly non-resonant regular singularities (i.e. with $\mu=\lambda_{2}-\lambda_{1} \notin$ $\mathbb{Z}$ ) have diagonalizable projective monodromy (conjugated to $f \mapsto$ $\left.e^{2 \pi i \mu} f\right)$.

(2) A regular singularity has non-diagonalizable projective monodromy if and only if its formal fundamental solution contains a logarithmic term.

TheOREM 1.4 (Analytic classification of regular singularities, $\nu=0$ ).

(1) Two LDEs (1.6) with regular singularities are analytically equivalent if and only if they have the same pair of formal invariants $\left\{\lambda_{1}, \lambda_{2}\right\}$ and their projective monodromies are conjugated, i.e. either they are both diagonalizable or both non-diagonalizable. 
(2) A LDE (1.6) with a regular singularity is always reducible. It is analytically equivalent to one of the following normal forms.

(a) Diagonalizable projective monodromy $\left(\lambda_{1} \neq \lambda_{2}\right)$ :

$$
\left(\delta_{0}-\lambda_{2}\right)\left(\delta_{0}-\lambda_{1}\right) y=0,
$$

whose basis of solutions is $y_{1}(x)=x^{\lambda_{1}}, y_{2}(x)=x^{\lambda_{2}}$.

(b) If $\lambda_{1}=\lambda_{2}$ (then the projective monodromy is non-diagonalizable):

$$
\left(\delta_{0}-\lambda_{1}\right)^{2} y=0,
$$

whose basis of solutions is $y_{1}(x)=x^{\lambda_{1}}, y_{2}(x)=x^{\lambda_{1}} \log x$.

(c) If $\lambda_{1}-\lambda_{2}=k \in \mathbb{Z}_{>0}$ and the projective monodromy is nondiagonalizable:

$$
\left(\delta_{0}-\lambda_{2}+k \frac{x^{k}}{1-x^{k}}\right)\left(\delta_{0}-\lambda_{1}\right) y=0,
$$

whose basis of solutions is $y_{1}(x)=x^{\lambda_{1}}, y_{2}(x)=x^{\lambda_{2}}+k x^{\lambda_{1}} \log x$. Alternatively, it is also analytically equivalent to

$$
\left(\delta_{0}-\lambda_{2}+k x^{k}\right)\left(\delta_{0}-\lambda_{1}\right) y=0 .
$$

\subsubsection{Non-resonant irregular singularities}

Proposition 1.5. - Two non-resonant irregular LDEs (1.6) are formally equivalent if and only if their pairs of formal invariants $\left\{\lambda_{1}(x) \delta_{\nu}^{-1}\right.$, $\left.\lambda_{2}(x) \delta_{\nu}^{-1}\right\}$ are in the same equivalence class. The formal transformation

$$
\widetilde{x}=\widehat{\phi}(x)=\sum_{j=1}^{+\infty} \phi^{(j)} x^{j}, \quad \widetilde{y}=\widehat{t}(x) \cdot y=\sum_{j=0}^{+\infty} t^{(j)} x^{j} \cdot y, \quad \phi^{(1)}, t^{(0)} \neq 0,
$$

between the two LDEs is then Borel $\nu$-summable (see Appendix for the definition), with singular directions $\arg (x)=\beta$ among those where $\Im\left(e^{-\nu \beta i}\left(\lambda_{2}^{(0)}-\right.\right.$ $\left.\left.\lambda_{1}^{(0)}\right)\right)=0$.

Assuming that their formal invariants are equal, then a formal transformation $\widehat{\phi}, \widehat{t}$ exists with $\widehat{\phi}(x)=x+t x^{\nu+1}+O\left(x^{\nu+2}\right)$ which is unique (up to $y \mapsto c y$ ) for any $t \in \mathbb{C}$.

In particular, the LDE is formally equivalent by means of a transformation (1.19) with $\widehat{\phi}(x)=x+O\left(x^{\nu+1}\right)$ to the following formal normal form

$$
\left(\delta_{\nu}-\lambda_{2}(\widetilde{x})-\frac{\delta_{\nu}\left(\lambda_{2}(\widetilde{x})-\lambda_{1}(\widetilde{x})\right)}{\lambda_{2}(\widetilde{x})-\lambda_{1}(\widetilde{x})}\right)\left(\delta_{\nu}-\lambda_{1}(\widetilde{x})\right) \widetilde{y}=0,
$$


i.e.

$$
\begin{aligned}
\widetilde{p}(\widetilde{x}) & =\lambda_{1}+\lambda_{2}+\delta_{\nu} \log \left(\lambda_{2}-\lambda_{1}\right), \\
\widetilde{q}(\widetilde{x}) & =-\lambda_{1} \lambda_{2}+\frac{1}{2} \delta_{\nu}\left(\lambda_{1}+\lambda_{2}\right)-\frac{\lambda_{1}+\lambda_{2}}{2} \delta_{\nu} \log \left(\lambda_{2}-\lambda_{1}\right), \\
\widetilde{\Delta}(\widetilde{x}) & =\left(\lambda_{2}-\lambda_{1}\right)^{2}-2 \delta_{\nu}^{2} \log \left(\lambda_{2}-\lambda_{1}\right)+\left(\delta_{\nu} \log \left(\lambda_{2}-\lambda_{1}\right)\right)^{2},
\end{aligned}
$$

whose basis of solutions is

$$
\widetilde{y}_{j}(\widetilde{x})=e^{\int \lambda_{j}(\widetilde{x}) \delta_{\nu}^{-1}}, \quad j=1,2 .
$$

Note that even though the factorization (1.20) is asymmetric, the equation itself is completely symmetric with respect to interchanging of $\lambda_{1}$ and $\lambda_{2}$.

The formal transformation $\widehat{\phi}(x)=x+O\left(x^{\nu+1}\right)$ is unique up to a composition with the flow of the vector field $\frac{1}{\lambda_{2}(x)-\lambda_{1}(x)} \delta_{\nu}$.

Up to an analytic change of coordinate $x \mapsto \phi(x)$, one can suppose that the pair $\left\{\lambda_{1}(x) \delta_{\nu}^{-1}, \lambda_{2}(x) \delta_{\nu}^{-1}\right\}$ is in the canonical form

$$
\lambda_{2}(x)-\lambda_{1}(x)=1+\mu x^{\nu} .
$$

Then the singular directions of $\widehat{\phi}(x), \widehat{t}(x)$ are $\beta_{l}=\frac{l}{\nu} \pi, l \in \mathbb{Z}$. Let $\phi_{\Omega_{l}}(x)$, $t_{\Omega_{l}}(x)$ be the Borel sums (see Appendix) of the formal transformation $\widehat{\phi}, \widehat{t}$, bounded and analytic on the sectors

$$
\Omega_{l}=\left\{\left|\arg x-\frac{2 l+1}{2 \nu} \pi\right|<\frac{\pi}{\nu}-\eta,|x|<\rho_{\eta}\right\}, \quad l \in \mathbb{Z}_{2 \nu},
$$

where $0<\eta<\frac{\pi}{2 \nu}$ is arbitrarily small, and $\rho_{\eta}>0$ depends on $\eta$. They transform the LDE to its formal normal form (1.20), which means that on each sector $\Omega_{l}$ the original LDE has a canonical basis of solutions

$$
y_{j, \Omega_{l}}=t_{\Omega_{l}}(x) \cdot \widetilde{y}_{j}\left(\phi_{\Omega_{l}}(x)\right), \quad j=1,2 .
$$

We can now define projective Stokes operators of the equation, corresponding to the projectivization of the Stokes matrices of the companion system, as the operators connecting the bases on neighboring sectors in the following way.

DeFINITION (Projective Stokes operators). - Let $f_{\Omega_{l}}(x):=\frac{y_{2, \Omega_{l}}(x)}{y_{1, \Omega_{l}}(x)}$, where $y_{j, \Omega_{l}}$ are the canonical sectoral solutions (1.21). The projective Stokes operators are the operators $\sigma_{\beta_{l}} \in \mathrm{PGL}_{2}(\mathbb{C})$ defined by

$$
\begin{aligned}
& f_{\Omega_{l-1}}(x)=\sigma_{\beta_{l}}\left(f_{\Omega_{l}}(x)\right), \quad \text { for } \arg (x)=\frac{l}{\nu} \pi, \quad l=1, \ldots, 2 \nu-1, \\
& f_{\Omega_{2 \nu}}\left(e^{2 \pi i} x\right) \cdot e^{-2 \pi i \mu}=\sigma_{\beta_{0}}\left(f_{\Omega_{0}}(x)\right), \text { for } \arg (x)=0 .
\end{aligned}
$$


They are of the form $\left\{\begin{array}{l}\sigma_{\beta_{l}}: f \mapsto f+s_{l} \text { if } l \text { is odd, i.e. when } e^{\int\left(\lambda_{2}-\lambda_{1}\right) \delta_{\nu}^{-1}} \text { is "exploding" as } x \rightarrow 0, \\ \sigma_{\beta_{l}}: f \mapsto \frac{f}{1+s_{l} f} \text { if } l \text { is even, i.e. when } e^{\int\left(\lambda_{2}-\lambda_{1}\right) \delta_{\nu}^{-1}} \text { is "flat" as } x \rightarrow 0 .\end{array}\right.$ Their collection $\left(\sigma_{\beta_{0}}, \ldots, \sigma_{\beta_{2 \nu-1}}\right)$ is well-defined up to simultaneous conjugation by a scalar multiplication (corresponding to the non-unicity of $\widehat{\phi}(x)$ ). It is extended to all $l \in \mathbb{Z}$ by

$$
\sigma_{\beta_{l+2 \nu}}(f)=e^{2 \pi i \mu} \sigma_{\beta_{l}}\left(e^{-2 \pi i \mu} f\right) .
$$

Remark. - The canonical pair of solutions $y_{1, \Omega_{l}}(x), y_{2, \Omega_{l}}(x)$ is up to multiplication by constants uniquely determined by their asymptotic behavior at the singular directions $\beta_{l}-\frac{\pi}{\nu}$ and $\beta_{l}+\frac{\pi}{\nu}$, one being flat at one direction the other being flat at the other direction.

DEFINITION (Rotational symmetries of the formal invariant). - Let $\left\{\lambda_{1}(x) \delta_{\nu}^{-1}, \lambda_{2}(x) \delta_{\nu}^{-1}\right\}$ be a pair of formal invariants in a canonical form. Let us define $G \subseteq \mathbb{Z}_{2 \nu}$ as the subgroup of the cyclic group consisting of the elements $g \in \mathbb{Z}_{2 \nu}$ such that the associated rotation $x \mapsto e^{\frac{g \pi i}{\nu}} x$ preserves the pair $\left\{\lambda_{1}(x) \delta_{\nu}^{-1}, \lambda_{2}(x) \delta_{\nu}^{-1}\right\}$. Since the pair of formal invariants in a canonical form is uniquely defined up to rotations from $\mathbb{Z}_{2 \nu}$, which commute with $G$, the group $G$ is well-defined.

For example, if the equation is trace-free, $\lambda_{1}(x)+\lambda_{2}(x)=0$, then either $G=\mathbb{Z}_{2 \nu}$ if $\mu=0$, or $G=2 \mathbb{Z}_{\nu}$ if $\mu \neq 0$.

THEOREM 1.6 (Analytic classification of non-resonant irregular singularities, $\nu>0)$.

(1) Two formally equivalent LDEs (1.6) with a non-resonant irregular singularity at the origin and the same pair of formal invariants in canonical form $\left\{\lambda_{1}(x) \delta_{\nu}^{-1}, \lambda_{2}(x) \delta_{\nu}^{-1}\right\}$ are analytically equivalent if and only if their respective collections of projective Stokes operators $\left(\sigma_{\beta_{l}}\right)_{l \in \mathbb{Z}}$ and $\left(\sigma_{\beta_{l}}^{\prime}\right)_{l \in \mathbb{Z}}$ are equivalent in the following sense: there exist $c \in \mathbb{C}^{*}$ and $g \in G$ such that

$$
\sigma_{\beta_{l}}^{\prime}=\iota^{g} \circ\left(\frac{1}{c} \sigma_{\beta_{l+g}}\right) \circ\left(c \iota^{g}\right) \text { for all } l \in \mathbb{Z},
$$

where $G \subseteq \mathbb{Z}_{2 \nu}$ is the symmetry group of the formal invariant and $\iota: f \mapsto \frac{1}{f}$.

(2) For every pair $\left\{\lambda_{1}(x) \delta_{\nu}^{-1}, \lambda_{2}(x) \delta_{\nu}^{-1}\right\}$ in canonical form and every collection of projective Stokes operators $\left(\sigma_{\beta_{0}}, \ldots, \sigma_{\beta_{2 \nu-1}}\right)$, there exists a LDE with non-resonant irregular singularity of a given formal class realizing them as its analytic invariants. 


\section{Proposition 1.7.}

(1) For a non-resonant irregular singularity the following are equivalent:

(a) The LDE (1.6) is reducible, i.e. of the form (1.14) for some $\alpha_{1}(x), \alpha_{2}(x)$.

(b) The LDE(1.6) has a "convergent solution" $y(x)=e^{\int \lambda(x) \delta_{\nu}^{-1}} t(x)$, where $\lambda(x)$ is one of the formal invariants and $t(x)$ is a convergent power series.

(c) The Riccati equation

$$
2 \delta_{\nu} r=r^{2}-\Delta(x)
$$

has an analytic solution $r(x)$. In this case $\alpha_{1}(x)=\frac{1}{2}(p(x)-$ $r(x)), \alpha_{2}(x)=\frac{1}{2}(p(x)+r(x))$.

(d) For either all odd or all even indices $l \in \mathbb{Z}$ the projective Stokes operators are trivial, $\sigma_{\beta_{l}}=\mathrm{id}$.

(2) For a non-resonant irregular singularity the following are equivalent:

(a) The LDE (1.6) is analytically equivalent to the formal normal form (1.20).

(b) The LDE (1.6) has a pair of "convergent solutions" $y(x)=$ $e^{\int \lambda_{j}(x) \delta_{\nu}^{-1}} t_{j}(x), j=1,2$, where $\left\{\lambda_{1}(x) \delta_{\nu}^{-1}, \lambda_{2}(x) \delta_{\nu}^{-1}\right\}$ are the formal invariants and $t_{j}(x)$ are convergent power series.

(c) The third order linear equation

$$
\delta_{\nu}^{3} h-\Delta(x) \delta_{\nu} h-\frac{1}{2}\left(\delta_{\nu} \Delta(x)\right) h=0 .
$$

has an analytic solution $h(x)$.

(d) The Riccati equation(1.22) has two different analytic solutions $r_{1}(x), r_{2}(x)$. In this case $h(x)=\frac{1}{r_{2}(x)-r_{1}(x)}$ is an analytic solution to (1.23).

(e) All the projective Stokes operators are trivial, $\sigma_{\beta_{l}}=\mathrm{id}$ for all $l \in \mathbb{Z}$.

The differential operator of the left-hand side of (1.23) is known as the second symmetric power of the operator $\delta_{\nu}^{2}-\frac{\Delta(x)}{4}[20, \S 3.1],[21, \S 2.3]$. If the LDE is reducible, and $r(x)$ is an analytic solution to (1.22), then the equation (1.23) can be factorized as

$$
\left(\delta_{\nu}-r(x)\right) \delta_{\nu}\left(\delta_{\nu}+r(x)\right) h=0 .
$$

Since in this case $r(0) \neq 0$, the formal/analytic solutions to (1.24) are also formal/analytic solutions to $\delta_{\nu}\left(\delta_{\nu}+r(x)\right) h=0$.

Remark. - If $y_{1}(x), y_{2}(x)$ are two linearly independent solutions to the LDE, $f(x)=\frac{y_{2}(x)}{y_{1}(x)}$, then $r=\frac{\delta_{\nu}^{2} f}{\delta f}=p-2 \frac{\delta_{\nu} y_{1}}{y_{1}}$ is a solution to (1.22), and 
$h=\frac{f}{\delta_{\nu} f}$ is a solution to (1.23). However, in general, they may not be analytic at 0 .

THEOREM 1.8 (Analytic normal forms when $\nu=1$ ).

(1) An irreducible LDE with a non-resonant irregular singularity at the origin of Poincaré rank $\nu=1$ is analytically equivalent to an LDE of the form

$$
\delta_{1}^{2} y-\left(p^{(0)}+x p^{(1)}\right) \delta_{1} y-\left(q^{(0)}+x q^{(1)}+x^{2} q^{(2)}\right) y,
$$

with $1=\Delta^{(0)}=\left(p^{(0)}\right)^{2}+4 q^{(0)}$ and $\mu=\frac{\Delta^{(1)}}{2}=p^{(0)} p^{(1)}+2 q^{(1)} \in \mathbb{C}$.

Two such equations are analytically equivalent if and only if

$$
\mu= \pm \widetilde{\mu}, \quad \text { and } \quad \cos \pi \sqrt{\Delta^{(2)}+1}=\cos \pi \sqrt{\widetilde{\Delta}^{(2)}+1},
$$

where $\Delta^{(2)}=\left(p^{(1)}\right)^{2}+4 q^{(2)}-2 p^{(1)}$.

(2) A reducible LDE (1.14) with a non-resonant irregular singularity at the origin of Poincaré rank $\nu=1$ with $\mu=\alpha_{2}^{(1)}-\alpha_{1}^{(1)} \notin \mathbb{Z}_{\leqslant 0}$ (hence with diagonalizable monodromy) is analytically equivalent to either

$$
\left(\delta_{1}-\lambda_{2}(x)\right)\left(\delta_{1}-\lambda_{1}(x)\right) y=0,
$$

with $\lambda_{2}(x)-\lambda_{1}(x)=1+\mu x$, or to $(1.20)$.

(3) A reducible LDE (1.14) with a non-resonant irregular singularity at the origin of Poincaré rank $\nu=1$ with $\mu=\alpha_{2}^{(1)}-\alpha_{1}^{(1)} \in \mathbb{Z}_{\leqslant 0}$ is analytically equivalent to either (1.25), which in this case is analytically equivalent to (1.20), if the monodromy is diagonalizable (scalar), or to

$$
\left(\delta_{1}-\lambda_{2}(x)+x^{2}\right)\left(\delta_{1}-\lambda_{1}(x)\right) y=0,
$$

with $\lambda_{2}(x)-\lambda_{1}(x)=1+\mu x$, if the monodromy is non-diagonalizable.

\subsubsection{Non-degenerate resonant irregular singularities}

Proposition 1.9. - Two non-degenerate resonant irregular LDEs (1.6) are formally equivalent if and only if their pairs of formal invariants $J_{0}^{\nu} p(x)$. $\delta_{\nu}^{-1}, J_{0}^{\nu} \Delta(x) \cdot \delta_{\nu}^{-2}$ are from the same equivalence class. The formal transformation

$$
\widetilde{x}=\widehat{\phi}(x)=\sum_{j=1}^{+\infty} \phi^{(j)} x^{j}, \quad \widetilde{y}=\widehat{t}(x) \cdot y=\sum_{j=0}^{+\infty} t^{(j)} x^{j} \cdot y, \quad \phi^{(1)}, t^{(0)} \neq 0,
$$

between the two LDEs is then Borel $\left(\nu-\frac{1}{2}\right)$-summable (see Appendix for the definition), with singular directions $\arg (x)=\beta$ among those where $\Im\left(e^{(1-2 \nu) \beta i} \frac{\mathrm{d} \Delta}{\mathrm{d} x}(0)\right)=0$. 
Assuming that their formal invariants are equal, then a unique formal transformation $\widehat{\phi}, \widehat{t}$ exists with $\widehat{\phi}(x)=x+O\left(x^{\nu+1}\right)$ and $\widehat{t}(0)=1$.

Suppose $J_{0}^{\nu} \Delta(x)=x$ and $J_{0}^{\nu} p(x)=P(x), P(0)=0$. Consider the formal normal form LDE (1.20) with $\lambda_{1}(x)+\lambda_{2}(x)=P(x)-\frac{1}{2} x^{\nu}, \lambda_{2}(x)-\lambda_{1}(x)=$ $x^{\frac{1}{2}}$, that is the LDE

$$
\delta_{\nu}^{2} \widetilde{y}-\widetilde{p}(x) \delta_{\nu} \widetilde{y}-\widetilde{q}(x) \widetilde{y}=0
$$

with

$$
\begin{aligned}
\widetilde{p}(x) & =P(x), \\
\widetilde{q}(x) & =\frac{1}{4}\left[x-P(x)^{2}+x^{\nu} P(x)-\left(\nu+\frac{1}{4}\right) x^{2 \nu}+2 \delta_{\nu} P(x)\right], \\
\widetilde{\Delta}(x) & =x+x^{\nu} P(x)-\left(\nu+\frac{1}{4}\right) x^{2 \nu},
\end{aligned}
$$

whose basis of solutions is $\widetilde{y}_{j}(x)=e^{\int \lambda_{j}(x) \delta_{\nu}^{-1}}, j=1,2$.

By the Proposition 1.9, the LDE is equivalent to (1.27) by a formal transformation $\widehat{\phi}(x), \widehat{t}(x)$, Borel $\left(\nu-\frac{1}{2}\right)$-summable except in the singular directions

$$
\beta_{l}=\frac{2 l}{2 \nu-1} \pi, \quad l \in \mathbb{Z} .
$$

Hence the LDE has a canonical basis of solutions

$$
y_{j, \Omega_{l}}=t_{\Omega_{l}}(x) \cdot \widetilde{y}_{j}\left(\phi_{\Omega_{l}}(x)\right), \quad j=1,2,
$$

where $\phi_{\Omega_{l}}(x), t_{\Omega_{l}}(x)$ are the Borel sums (see Appendix), bounded and analytic on the sectors

$$
\Omega_{l}=\left\{\left|\arg x-\frac{2 l+1}{2 \nu-1} \pi\right|<\frac{2 \pi}{2 \nu-1}-\eta,|x|<\rho_{\eta}\right\}, \quad l \in \mathbb{Z}_{2 \nu-1},
$$

where $0<\eta<\frac{\pi}{2 \nu-1}$ is arbitrarily small, and $\rho_{\eta}>0$ depends on $\eta$.

The projective Stokes operators $\sigma_{\beta_{l}} \in \mathrm{PGL}_{2}(\mathbb{C})$ are now defined as before

$$
\begin{aligned}
f_{\Omega_{l-1}}(x) & =\sigma_{\beta_{l}}\left(f_{\Omega_{l}}(x)\right), & \text { for } \arg (x) & =\frac{l}{\nu} \pi, \quad l=1, \ldots, 2 \nu-1, \\
f_{\Omega_{2 \nu}}\left(e^{2 \pi i} x\right)^{-1} & =\sigma_{\beta_{0}}\left(f_{\Omega_{0}}(x)\right), & \text { for } \arg (x) & =0 .
\end{aligned}
$$

where $f_{\Omega_{l}}(x):=\frac{y_{2, \Omega_{l}}(x)}{y_{1, \Omega_{l}}(x)}$. Their definition is extended to all $l \in \mathbb{Z}$ by

$$
\sigma_{\beta_{l+2 \nu}}=\iota \circ \sigma_{\beta_{l}} \circ \iota, \quad \text { where } \iota: f \mapsto \frac{1}{f} .
$$

They are of the form

$$
\left\{\begin{array}{l}
\sigma_{\beta_{l}}: f \mapsto f+s_{l} \text { when } e^{\int\left(\lambda_{2}-\lambda_{1}\right) \delta_{\nu}^{-1}} \text { is "exploding" as } x \rightarrow 0, \\
\sigma_{\beta_{l}}: f \mapsto \frac{f}{1+s_{l} f} \text { when } e^{\int\left(\lambda_{2}-\lambda_{1}\right) \delta_{\nu}^{-1}} \text { is "flat" as } x \rightarrow 0 .
\end{array}\right.
$$


Definition (Rotational symmetries of the formal invariant). - Suppose $J_{0}^{\nu} \Delta(x)=x, J_{0}^{\nu} p(x)=P(x)$. Let us define $G \subseteq \mathbb{Z}_{2 \nu-1}$ as the subgroup of the cyclic group consisting of the elements $g \in \mathbb{Z}_{2 \nu-1}$ such that the associated rotation $x \mapsto e^{\frac{g 2 \pi i}{2 \nu-1}} x$ preserves the differential form $P(x) \delta_{\nu}^{-1}$. Since $P(x) \delta_{\nu}^{-1}$ is uniquely defined up to rotations from $\mathbb{Z}_{2 \nu-1}$, which commute with $G$, the group $G$ is well-defined.

THEOREOM 1.10 ([Analytic classification of non-degenerate resonant irregular singularities, $\nu>0)$.

(1) Two formally equivalent LDEs (1.6) with a non-degenerate resonant irregular singularity at the origin and the same pair of formal invariants in canonical form $x \delta_{\nu}^{-2}, P(x) \delta_{\nu}^{-1}$ are analytically equivalent if and only if their respective collections of projective Stokes operators $\left(\sigma_{\beta_{l}}\right)_{l \in \mathbb{Z}}$ and $\left(\sigma_{\beta_{l}}^{\prime}\right)_{l \in \mathbb{Z}}$ are equivalent in the following sense: there exist $g \in G$ such that

$$
\sigma_{\beta_{l}}^{\prime}=\iota^{g} \circ \sigma_{\beta_{l+g}} \circ \iota^{g} \quad \text { for all } l \in \mathbb{Z},
$$

where $G \subseteq \mathbb{Z}_{2 \nu-1}$ is the symmetry group of the formal invariant and $\iota: f \mapsto \frac{1}{f}$.

(2) For every pair of formal invariants $x \delta_{\nu}^{-2}, P(x) \delta_{\nu}^{-1}$ in canonical form and every collection of projective Stokes operators, there exists a $L D E$ with non-degenerate resonant irregular singularity of given formal class realizing them as its analytic invariants.

TheOREM 1.11 (Analytic normal forms when $\nu=1$ ). - LDE with a non-degenerate resonant irregular singularity at the origin of Poincaré rank $\nu=1$ is analytically equivalent to an $L D E$ of the form

$$
\delta_{1}^{2} y-p^{(1)} x \delta_{1} y-\left(\frac{1}{4} x+x^{2} q^{(2)}\right) y,
$$

with $\Delta(x)=x+x^{2} \Delta^{(2)}$, with $\Delta^{(2)}=\left(p^{(1)}\right)^{2}+4 q^{(2)}-2 p^{(1)}$. Two such equations are analytically equivalent if and only if

$$
p^{(1)}=\widetilde{p}^{(1)}, \quad \text { and } \quad \cos \pi \sqrt{1+\Delta^{(2)}}=\cos \pi \sqrt{1+\widetilde{\Delta}^{(2)}} .
$$

\subsection{Meromorphic classification}

If the transformation (1.5) is meromorphic with $t(x)=x^{m} u(x), m \in \mathbb{Z}$, $u(0) \neq 0$, then from (1.9)-(1.11) one can see that

$$
p(x)=\psi(x) \cdot \widetilde{p}(\phi(x))-2 m x^{\nu}+O\left(x^{2 \nu+1}\right), \quad \Delta(x)=\psi(x)^{2} \cdot \widetilde{\Delta}(\phi(x))+O\left(x^{\nu+1}\right) .
$$


In the regular or non-resonant irregular case this means that $\lambda_{1,2}(x)=$ $\psi(x) \widetilde{\lambda}_{1,2}(\phi(x))-m x^{\nu}+O\left(x^{\nu+1}\right)$, i.e. that equivalence class of the pair of formal invariants $\left\{\lambda_{1}(x) \delta_{\nu}^{-1}, \lambda_{2}(x) \delta_{\nu}^{-1}\right\}$ is shifted by $-m x^{\nu} \delta_{\nu}^{-1}$.

THEOREM 1.12 (Meromorphic classification). — Two LDEs (1.6) with either regular or non-degenerate irregular singularities are meromorphically equivalent if and only if they have the same equivalence class of formal invariants $J_{0}^{\nu} \Delta(x) \cdot \delta_{\nu}^{-2}, J_{0}^{\nu} p(x) \cdot \delta_{\nu}^{-1}$ up to a shift $J_{0}^{\nu} p(x) \cdot \delta_{\nu}^{-1} \mapsto J_{0}^{\nu} p(x) \cdot$ $\delta_{\nu}^{-1}+m x^{\nu} \delta_{\nu}^{-1}, m \in \mathbb{Z}$, and

(1) if regular: their monodromies are conjugated (i.e. they are both either diagonalizable or non-diagonalizable),

(2) if non-resonant irregular: their collections of Stokes operators are equivalent in the sense of Theorem 1.6,

(3) if non-degenerate resonant irregular: their collections of Stokes operators are equivalent in the sense of Theorem 1.10.

Let us remark that, unlike for systems, conjugation of monodromies of regular singularities alone does not suffice to produce meromorphic equivalence.

\subsection{Lie symmetries}

For non-resonant singularities of differential systems there is a canonical diagonal formal normal form

$$
\delta_{\nu} u=\left(\begin{array}{cc}
\lambda_{1}(x) & 0 \\
0 & \lambda_{2}(x)
\end{array}\right) u
$$

which is integrable in terms of elementary functions with fundamental solution matrix $\left(\begin{array}{cc}e^{\int \lambda_{1}(x) \delta_{\nu}^{-1}} & 0 \\ 0 & e^{\int \lambda_{2}(x) \delta_{\nu}^{-1}}\end{array}\right)$. Correspondingly, the analytic class of this normal form system is the one that has the largest possible Lie algebra of analytic infinitesimal symmetries (see [5]) of all the systems within the formal class. It turns out that the same holds also for non-resonant irregular LDEs (1.6): they are analytically equivalent to their formal normal form (1.20) if and only their Lie algebra of linear analytic point symmetries is the largest possible (Theorem 1.13 below).

Let us recall that an infinitesimal linear symmetry of a LDE (1.1) is a vector field

$$
Y=g(x) \frac{\partial}{\partial x}+f(x) y \frac{\partial}{\partial y},
$$


whose second jet prolongation $\operatorname{pr}^{(2)} Y$ leaves the surface $y_{x x}+a_{1}(x) y_{x}+$ $a_{0}(x) y=0$ invariant. This is equivalent $[7$, p. 350$]$ to ask that

$$
\left[X, \operatorname{pr}^{(1)} Y\right]=\alpha(x) X, \quad \text { for some function } \alpha(x),
$$

where

$$
X=\frac{\partial}{\partial x}+y_{x} \frac{\partial}{\partial y}+\left(a_{1}(x) y_{x}+a_{0}(x) y\right) \frac{\partial}{\partial y_{x}},
$$

and $\operatorname{pr}^{(1)} Y$ is the first jet prolongation of $Y$ :

$$
\operatorname{pr}^{(1)} Y=g(x) \frac{\partial}{\partial x}+f(x) y \frac{\partial}{\partial y}+\left(\frac{\mathrm{d} f}{\mathrm{~d} x}(x) y+f(x) y_{x}-\frac{\mathrm{d} g}{\mathrm{~d} x}(x) y_{x}\right) \frac{\partial}{\partial y_{x}} .
$$

THEOREM 1.13. - The infinitesimal linear symmetries of a LDE (1.6) are of the form

$$
Y=h(x) \delta_{\nu}+\frac{1}{2}\left(c+\delta_{\nu} h(x)+p(x) h(x)\right) y \frac{\partial}{\partial y},
$$

where $c \in \mathbb{C}$, and $h(x)$ is a solution of (1.23). The Lie algebra of analytic infinitesimal linear symmetries of

(1) a regular singularity:

(a) strictly non-resonant with $\lambda_{1}-\lambda_{2} \notin \mathbb{Z} \backslash\{0\}$ in the normal form (1.15) is generated by

$$
y \frac{\partial}{\partial y}, \quad \delta_{0},
$$

(b) resonant with $\lambda_{1}-\lambda_{2}=k \in \mathbb{Z}_{>0}$ and trivial projective monodromy, in the normal form (1.15), is generated by

$$
y \frac{\partial}{\partial y}, \quad \delta_{0}, \quad x^{k}\left(\delta_{0}+\lambda_{1} y \frac{\partial}{\partial y}\right),
$$

(c) resonant with $\lambda_{1}-\lambda_{2}=k \in \mathbb{Z}_{>0}$ and non-diagonalizable projective monodromy, in the normal form (1.17),

$$
y \frac{\partial}{\partial y}, \quad \frac{x^{k}}{1-x^{k}}\left(\delta_{0}+\lambda_{1} y \frac{\partial}{\partial y}\right),
$$

(2) a non-resonant irregular singularity of Poincaré rank $\nu>0$ :

(a) in the normal form (1.20) is generated by

$$
y \frac{\partial}{\partial y}, \quad \frac{1}{\lambda_{2}(x)-\lambda_{1}(x)}\left(\delta_{\nu}+\frac{\lambda_{1}(x)+\lambda_{2}(x)}{2} y \frac{\partial}{\partial y}\right),
$$

(b) not analytically equivalent to (1.20) is generated only by

$$
y \frac{\partial}{\partial y} \text {. }
$$


(3) a non-degenerate resonant irregular singularity of Poincaré rank $\nu>$ 0 is generated only by

$$
y \frac{\partial}{\partial y} \text {. }
$$

Let us remark that for non-singular LDEs the Lie algebra of analytic linear infinitesimal symmetries is of maximal dimension 4 , namely for $\frac{\mathrm{d}^{2} y}{\mathrm{~d} x^{2}}=$ 0 it is generated by

$$
y \frac{\partial}{\partial y}, \quad \frac{\partial}{\partial x}, \quad x \frac{\partial}{\partial x}, \quad x\left(x \frac{\partial}{\partial x}+y \frac{\partial}{\partial y}\right),
$$

while the Lie algebra of all analytic infinitesimal symmetries, i.e. infinitesimal point symmetries of the form $Y=G(x, y) \partial_{x}+F(x, y) \partial_{y}$, is of dimension 8 (and is well known to be isomorphic to $\mathfrak{s l}_{3}(\mathbb{C})$ ).

\section{Proofs}

We will review and adapt to our needs some basics of the theory of singularities of linear differential systems which can be found in some form in most standard references, e.g. in $[1,2,3,9,15,21,22]$. In the case of regular singularities the analytic classification agrees with a formal one, and in the case of non-resonant irregular singularities the analytic modulus consists of a set of formal invariants and of a conjugacy equivalence class of a collection of Stokes matrices.

Proof of Theorem 1.1. - Follows from Theorems 1.4, 1.6 and 1.10.

\section{Regular singular points}

If $\nu=0$, the singular point is of Fuchsian kind and, according to the general theory [9, Theorem 16.16], the companion system (1.7) is analytically equivalent by a gauge transformation $v=T(x) \widetilde{v}$ to a normal form

$$
\delta_{0} \widetilde{v}=\left(\begin{array}{cc}
\lambda_{1} & \epsilon x^{\lambda_{1}-\lambda_{2}} \\
0 & \lambda_{2}
\end{array}\right) \widetilde{v},
$$

where $\epsilon \in\{0,1\}$ and $\epsilon \neq 0$ only if $\lambda_{1}-\lambda_{2} \in \mathbb{Z}_{\geqslant 0}$. Therefore the system (1.7) possesses a fundamental solution matrix $V(x)=T(x)\left(\begin{array}{cc}x^{\lambda_{1}} & \epsilon x^{\lambda_{1}} \log x \\ 0 & x^{\lambda_{2}}\end{array}\right)$, where $T(x)=\left(T_{i j}(x)\right)$ is analytic and

$$
T(0)= \begin{cases}\left(\begin{array}{cc}
1 & 1 \\
\lambda_{1} & \lambda_{2}
\end{array}\right) & \text { if } \lambda_{1} \neq \lambda_{2}, \\
\left(\begin{array}{cc}
1 & 0 \\
\lambda_{1} & 1
\end{array}\right) & \text { if } \lambda_{1}=\lambda_{2} \text { in which case } \epsilon=1 .\end{cases}
$$


The complete analytic invariant of the system is given by the pair $\left\{\lambda_{1}, \lambda_{2}\right\}$ and by $\epsilon \in\{0,1\}$. The monodromy matrix $M$ of this fundamental solution, $V\left(e^{2 \pi i} x\right)=V(x) M$, is then given by $M=\left(\begin{array}{cc}e^{2 \pi i \lambda_{1}} & \epsilon 2 \pi i e^{2 \pi i \lambda_{1}} \\ 0 & e^{2 \pi i \lambda_{2}}\end{array}\right)$.

The LDE has therefore a solution basis

$$
y_{1}(x)=T_{11}(x) x^{\lambda_{1}}, \quad y_{2}(x)=T_{12}(x) x^{\lambda_{2}}+\epsilon T_{11}(x) x^{\lambda_{1}} \log x .
$$

Proof of Lemma 1.3. - By the above, the strict non-resonance condition $\lambda_{1}-\lambda_{2} \notin \mathbb{Z}$ means $\epsilon=0$. The diagonalizability of the monodromy $M$ is equivalent to $\epsilon=0$, which is equivalent to the absence of $\log x$ in (2.2).

Proof of Theorem 1.4. - The statement (1) is a corollary of (2).

(2a) Regular singularity with diagonalizable monodromy. - By the above considerations, the $\operatorname{LDE}(1.6)$ has a solution basis (2.2) with $\epsilon=0$, where $T_{1 j}(x)$ is an analytic germ with $T_{1 j}(0)=1$. We are looking for an analytic transformation $\widetilde{x}=\phi(x), \widetilde{y}=t(x) y(1.5)$, such that

$$
T_{1 j}(x) x^{\lambda_{j}}=t(x) \cdot \phi(x)^{\lambda_{j}}, j=1,2 .
$$

Writing $\phi(x)=x(1+g(x)), g(0)=0$, then $g(x)$ is a solution to

$$
\log \left(\frac{T_{12}}{T_{11}}\right)(x)=\left(\lambda_{2}-\lambda_{1}\right) \log (1+g(x)),
$$

where the right-hand side is an analytic function of $x$ and $g$ whose derivative with respect to $g$ at $(x, g)=0$ is $\lambda_{2}-\lambda_{1} \neq 0$, so it has by the implicit function theorem a unique analytic solution $g(x)$ with $g(0)=0$. Then also $t(x)=T_{11}(x)(1+g(x))^{-\lambda_{1}}$ is an analytic germ, $t(0)=1$.

(2b) Regular singularity with $\lambda_{1}=\lambda_{2}=: \lambda$. - The LDE (1.6) has a solution basis $(2.2)$ with $\epsilon=1$, where $T_{11}(0)=1, T_{12}(0)=0$. The transformation equation we want to solve is

$T_{11}(x) x^{\lambda}=t(x) \cdot \phi(x)^{\lambda}, \quad T_{12}(x) x^{\lambda}+T_{11}(x) x^{\lambda} \log x=t(x) \cdot \phi(x)^{\lambda} \log \phi(x)$.

Writing $\phi(x)=x(1+g(x)), g(0)=0$, then

$$
\frac{T_{12}}{T_{11}}(x)=\log (1+g(x)), \quad \text { hence } g(x)=e^{\frac{T_{12}}{T_{11}}(x)}-1,
$$

and $t(x)=T_{11}(x)(1+g(x))^{-\lambda}, t(0)=1$.

(2c) Regular singularity with non-diagonalizable monodromy, $\lambda_{1}-\lambda_{2}=$ $k \in \mathbb{Z}_{>0}$. - The $\operatorname{LDE}(1.6)$ has a solution basis (2.2) with $\epsilon=1$, where $T_{11}(0)=T_{12}(0)=1$. The transformation equation we want to solve is

$$
\begin{aligned}
T_{11}(x) x^{\lambda_{1}} & =t(x) \cdot \phi(x)^{\lambda_{1}}, \\
k\left(T_{12}(x) x^{\lambda_{2}}+T_{11}(x) x^{\lambda_{1}} \log x\right) & =t(x) \cdot\left(\phi(x)^{\lambda_{2}}+k \phi(x)^{\lambda_{1}} \log \phi(x)\right) .
\end{aligned}
$$


Writing $\phi(x)=c x(1+g(x)), g(0)=0$, let $c=k^{-\frac{1}{k}}$, then $g(x)$ is a solution to

$$
\frac{T_{12}}{T_{11}}(x)=(1+g(x))^{-k}+x^{k} \log (1+g(x))-\frac{x^{k}}{k} \log k .
$$

The derivative of the right side with respect to $g$ at $(x, g)=0$ is $\lambda_{2}-\lambda_{1}=-k$, therefore the equation has a unique analytic solution $g(x)$ with $g(0)=0$. Then $t(x)=T_{11}(x) c^{-\lambda_{1}}(1+g(x))^{-\lambda_{1}}, t(0)=c^{-\lambda_{1}}$.

\section{Non-resonant irregular singular points}

Let $\lambda_{j}(x)=\lambda_{j}^{(0)}+\ldots+\lambda_{j}^{(\nu)} x^{\nu}, j=1,2$, be modulo $x^{\nu+1}$ the roots of the characteristic polynomial $\lambda^{2}-p(x) \lambda-q(x)=0$. If $\nu>0$ and $\lambda_{1}(0) \neq \lambda_{2}(0)$, then the singular point is non-resonant irregular and, according to the general theory $[9, \S 20]$, the companion system (1.7) possesses a formal fundamental solution matrix $\widehat{V}(x)=\widehat{T}(x) e^{\int\left(\begin{array}{cc}\lambda_{1} & 0 \\ 0 & \lambda_{2}\end{array}\right) \delta_{\nu}^{-1}}$, where $\widehat{T}(x)=\left(\widehat{T}_{i j}(x)\right)$ is a formal power series, $\widehat{T}(0)=\left(\begin{array}{cc}1 & 1 \\ \lambda_{1}^{(0)} & \lambda_{2}^{(0)}\end{array}\right)$. Correspondingly, the LDE has a formal solution basis

$$
\widehat{y}_{1}(x)=\widehat{T}_{11}(x) e^{\int \lambda_{1}(x) \delta_{\nu}^{-1}}, \quad \widehat{y}_{2}(x)=\widehat{T}_{12}(x) e^{\int \lambda_{2}(x) \delta_{\nu}^{-1}} .
$$

A complete formal invariant of the system (1.7) with respect to formal gauge transformations $v \mapsto T(x) v$ (1.4) is formed by the pair of meromorphic 1forms $\left\{\lambda_{1}(x) \delta_{\nu}^{-1}, \lambda_{2}(x) \delta_{\nu}^{-1}\right\}$. If one allows also for transformations $x \mapsto \phi(x)$, then it is always possible to transform analytically the pair to a canonical form where $\left(\lambda_{2}(x)-\lambda_{1}(x)\right) \delta_{\nu}^{-1}=\left(1+\mu x^{\nu}\right) \delta_{\nu}^{-1}$, where $\mu$ is well-defined up to the \pm sign.

A Stokes direction (also known as separating direction) $\alpha \in \mathbb{R}$ is defined by $\Re\left(e^{-\nu \alpha}\left(\lambda_{2}^{(0)}-\lambda_{1}^{(0)}\right)\right)=0$, and an anti-Stokes direction (also known as singular direction) $\beta \in \mathbb{R}$ is defined by $\Im\left(e^{-\nu \beta}\left(\lambda_{2}^{(0)}-\lambda_{1}^{(0)}\right)\right)=0$. After the normalization $\lambda_{2}^{(0)}-\lambda_{1}^{(0)}=1$, this means $\alpha \in \frac{\pi}{\nu} \mathbb{Z}, \beta \in \frac{\pi}{2 \nu}+\frac{\pi}{\nu} \mathbb{Z}$. Let $\left\{\alpha_{l}\right\}_{l \in \mathbb{Z}}$, resp. $\left\{\beta_{l}\right\}_{l \in \mathbb{Z}}$, be all the Stokes directions, resp. anti-Stokes directions in their order, $\alpha_{l+2 \nu}=\alpha_{l}+2 \pi$. By a classical theorem of Horn, Trjitzinsky, Hukuhara, Turittin, and others [9, Theorem 20.16], the formal power series $\widehat{T}(x)$ is asymptotic to a unique bounded analytic matrix-valued function $T_{\alpha_{l}}(x)$ on every open sector $\Omega_{l}$ covering exactly one Stokes direction $\arg (x)=$ $\alpha_{l}$. Let $\Omega_{2 \nu}=\Omega_{0}, \ldots, \Omega_{2 \nu-1}$ be a cyclic covering by such sectors of a pointed neighborhood of the origin, such that the intersection of two neighboring sectors covers exactly one anti-Stokes direction (when considered on the Riemann surface of $\log x$ ), let $T_{\alpha_{2 \nu}}=T_{\alpha_{0}} n \ldots, T_{\alpha_{2 \nu-1}}$ be the associated 
sectorial transformations, and let $V_{\alpha_{l}}(x)=T_{\alpha_{l}}(x) e^{\int\left(\begin{array}{cc}\lambda_{1}(x) & 0 \\ 0 & \lambda_{2}(x)\end{array}\right) \delta_{\nu}^{-1}}$ be the sectorial fundamental matrix solutions, $V_{\alpha_{l}+2 \pi}(x)=V_{\alpha_{l}}(x) e^{2 \pi i\left(\begin{array}{cc}\lambda_{1}^{(\nu)} & 0 \\ 0 & \lambda_{2}^{(\nu)}\end{array}\right)}$. For each anti-Stokes direction $\beta$ the Stokes matrix $S t_{\beta}$ is defined by

$$
V_{\beta-\frac{\pi}{2 \nu}}=V_{\beta+\frac{\pi}{2 \nu}} S t_{\beta} .
$$

The collection of the Stokes matrices $\left\{S t_{\beta_{0}}, \ldots, S t_{\beta_{2 \nu-1}}\right\}$ modulo simultaneous conjugation by diagonal matrices is a complete analytic invariant of the system (1.7) with given formal invariants (sometimes called MalgrangeSibuya modulus) [9, Theoerm 20.21].

Proof of Proposition 1.5. - By the above considerations, the LDE (1.6) has a formal solution basis $(2.3)$, where $\widehat{T}_{1 j}(x)$ is formal power series that is Borel $\nu$-summable, $\widehat{T}_{1 j}(0) \neq 0$. After an eventual analytic change of the $x$-variable, we can suppose that the formal invariants $\left\{\lambda_{1}(x) \delta_{\nu}^{-1}, \lambda_{2}(x) \delta_{\nu}^{-1}\right\}$ are in a canonical form with $\lambda_{2}(x)-\lambda_{1}(x)=1+\mu x^{\nu}$. We are looking for a formal transformation

$$
\widetilde{x}=\widehat{\phi}(x)=x\left(1+x^{\nu} \widehat{g}(x)\right), \quad \widetilde{y}=\widehat{t}(x) y,
$$

such that

$$
\widehat{T}_{1 j}(x) e^{\int \lambda_{j}(x) \delta_{\nu}^{-1}}=\widehat{t}(x) \cdot\left(e^{\int \lambda_{j} \delta_{\nu}^{-1}}\right) \circ \widehat{\phi}(x), j=1,2 .
$$

Therefore $\widehat{g}(x)$ is a solution to

$$
\log \left(\frac{\widehat{T}_{12}}{\widehat{T}_{11}}\right)(x)=\frac{1}{\nu x^{\nu}}-\frac{1}{\nu x^{\nu}\left(1+x^{\nu} \widehat{g}\right)^{\nu}}+\mu \log \left(1+x^{\nu} \widehat{g}\right),
$$

where the right-hand side is an analytic function of $x$ and $g$ whose derivative with respect to $\widehat{g}$ at $x=0$ is $\lambda_{2}^{(0)}-\lambda_{1}^{(0)}=1$, so by the formal implicit function theorem it has a unique formal solution $\widehat{g}(x)$ with $\widehat{g}(0)=\log \left(\frac{\hat{T}_{12}(0)}{\hat{T}_{11}(0)}\right)$. Since $\widehat{\phi}(x)=x+O\left(x^{\nu+1}\right)$ then also $\widehat{t}(x)$ is a formal power series, $\widehat{t}(0)=\widehat{T}_{11}(0) e^{-\lambda_{1}^{(0)} \hat{g}(0)}$.

One can also solve the equation on the sectors $\Omega_{\alpha}$ (see Proposition 2.2 in the Appendix) and deduce that $\widehat{\phi}(x), \widehat{t}(x)$ are Borel $\nu$-summable in the same directions as is the pair $\left(\widehat{T}_{11}(x), \widehat{T}_{12}(x)\right)$.

\section{Proof of Theorem 1.6. -}

(1). Analytic equivalence. - Let us show that if two LDEs (1.6) with non-resonant irregular singularity have their companion systems (1.7) analytically equivalent, than so are the equations. After an analytic change of $x$ we can assume that the formal invariants $\left\{\lambda_{1}(x) \delta_{\nu}^{-1}, \lambda_{2}(x) \delta_{\nu}^{-1}\right\}$ are in the 
canonical form and are the same for the two systems. Up to right multiplication of $\widehat{T}(x)$ by a constant diagonal matrix, we can also suppose that their collections of Stokes matrices agree. Therefore, for a singular direction $\beta$ :

$$
\begin{aligned}
& T_{1 j, \beta-}(x)=T_{1 j, \beta+}(x)+s_{\beta} T_{1 i, \beta+}(x) e^{\int\left(\lambda_{i}(x)-\lambda_{j}(x)\right) \delta_{\nu}^{-1}}, \\
& T_{1 i, \beta-}(x)=T_{1 i, \beta+}(x), \\
& \widetilde{T}_{1 j, \beta-}(x)=\widetilde{T}_{1 j, \beta+}(x)+s_{\beta} \widetilde{T}_{1 i, \beta+}(x) e^{\int\left(\lambda_{i}(x)-\lambda_{j}(x)\right) \delta_{\nu}^{-1}}, \\
& \widetilde{T}_{1 i, \beta-}(x)=\widetilde{T}_{1 i, \beta+}(x),
\end{aligned}
$$

where $s_{\beta}$ is the Stokes multiplier on the position $(j, i)$ of $S t_{\beta}$, with $(j, i)=$ $(1,2)$ or $(2,1)$ depending on $\beta$ such that $e^{\int\left(\lambda_{i}(x)-\lambda_{j}(x)\right)}$ is flat when $x \rightarrow 0$, $\arg x=\beta$. The conjugation equations to solve are

$$
T_{1 l, \beta \pm}(x) e^{\int \lambda_{l}(x) \delta_{\nu}^{-1}}=t_{\beta \pm}(x) \cdot\left(\widetilde{T}_{1 l, \beta \pm} e^{\int \lambda_{l} \delta_{\nu}^{-1}}\right) \circ \phi_{\beta \pm}(x), \quad l=1,2 .
$$

Comparing the above expressions we see that on the intersection sector $\Omega_{\beta+\frac{\pi}{2 \nu}} \cap \Omega_{\beta-\frac{\pi}{2 \nu}}$ both $\phi_{\beta-}(x)$ and $\phi_{\beta \pm+}(x)$ solve the same functional equation

$$
\frac{T_{1 j, \beta \pm}}{T_{1 i, \beta \pm}} e^{\int \lambda_{j}-\lambda_{i} \delta_{\nu}^{-1}}=\frac{\widetilde{T}_{1 j, \beta \pm}}{\widetilde{T}_{1 i, \beta \pm}} e^{\int \lambda_{j}-\lambda_{i} \delta_{\nu}^{-1}} \circ \phi_{\beta \pm},
$$

hence they shall be equal if the existence and the unicity of a bounded sectorial solution is ensured, which means that they will all glue up together to form an analytic germ $\phi(x)$. Writing $\phi_{\beta \pm}(x)=x\left(1+x^{\nu} g_{\beta \pm}(x)\right)$, the conjugation equation becomes

$$
\begin{aligned}
\left.\log \left(\frac{T_{1 j, \beta \pm}}{T_{1 i, \beta \pm}}\right)(x)=\log \left(\frac{\widetilde{T}_{1 j, \beta \pm}}{\widetilde{T}_{1 i, \beta \pm}}\right)\left(x+x^{\nu+1} g_{\beta \pm}\right)\right)+\frac{1}{\nu x^{\nu}} & \\
- & \frac{1}{\nu x^{\nu}\left(1+x^{\nu} g_{\beta \pm}\right)^{\nu}}+\mu \log \left(1+x^{\nu} g_{\beta \pm}(x)\right),
\end{aligned}
$$

which by virtue of Proposition 2.2 in the Appendix, has a unique bounded analytic solution $g_{\beta \pm}(x)$ on $\Omega_{\beta \pm \frac{\pi}{2 \nu}}$ satisfying $g_{\beta \pm}(0)=\log \left(\frac{T_{1 j}(0) \tilde{T}_{1 i}(0)}{T_{1 i}(0) \tilde{T}_{1 j}(0)}\right)$. And similarly with $t_{\beta+}=t_{\beta-}$.

(2). Realization. - Given $\nu>1$, formal invariants $\lambda_{1}(x), \lambda_{2}(x)$, and a collection of projective Stokes operators, we want to show that there exists an equation (1.6) of which they are analytic invariants.

By the Birkhoff-Malgrange-Sibuya theorem ([4], [15], [22], [9, Theorem 20.22], [1, Theorem 4.5.1]) there exists a differential system $\delta_{\nu} v=A(x) v$ with the given formal invariants realizing the associated Stokes matrices as its analytic invariants. Under the non-resonance condition one may as well assume that $A(0)=\left(\begin{array}{cc}\lambda_{1}(0) & 1 \\ 0 & \lambda_{2}(0)\end{array}\right)$. Writing $A=\left(A_{i j}\right)$, then it is easy to 
verify that the system satisfied by $\widetilde{v}=\left(\begin{array}{cc}1 & 0 \\ A_{11}(x) & A_{12}(x)\end{array}\right) v$ is of the companion form.

Another possibility is to prove the realization theorem directly using the Ahlfors-Bers theorem. For the sake of completeness let us give a rough sketch of this second approach, following the ideas of Malgrange [15, 16]. Let $f(x)=e^{\int\left(\lambda_{2}-\lambda_{1}\right) \delta_{\nu}^{-1}(x)}$. For an anti-Stokes direction $\beta$ and a projective Stokes operator $\sigma_{\beta}$, let $\psi_{\beta}(x) \sim x+O\left(e^{-\frac{c}{|x|^{\nu}}}\right.$ ) (with some $c>0$ ) be defined by solving the equation $\sigma_{\beta} \circ f(x)=f \circ \psi_{\beta}(x)$ on a small sector bisected by $\beta, x \in \Omega_{\beta+\frac{\pi}{2 \nu}} \cap \Omega_{\beta-\frac{\pi}{2 \nu}}$, where $\Omega_{\alpha}=\left\{|\arg x-\alpha|<\frac{\pi}{\nu}-\eta,|x|<\rho\right\}$ for some $0<\eta<\frac{\pi}{2 \nu},|\rho|>0$. We then want to find bounded analytic sectorial maps $\phi_{\alpha}(x)=x+O\left(x^{2}\right), x \in \Omega_{\alpha}$ that solve the cohomological equation

$$
\psi_{\beta}=\phi_{\beta-\frac{\pi}{2 \nu}} \circ \phi_{\beta+\frac{\pi}{2 \nu}}^{\circ-1} .
$$

Since $f \circ \phi_{\beta-\frac{\pi}{2 \nu}}=\sigma_{\beta} \circ\left(f \circ \phi_{\beta+\frac{\pi}{2 \nu}}\right)$ are related by a projective transformation, they define the same $\Delta(x)=-2 \mathcal{S}_{\nu}\left(f \circ \phi_{\alpha}\right)$ for all $\alpha$, which is therefore analytic on a neighborhood of 0 , and the equation (1.6) with $p(x)=\lambda_{1}(x)+$ $\lambda_{2}(x)$ and $q(x)=\frac{1}{4}\left(\Delta(x)-p(x)^{2}+2 \delta_{\nu} p(x)\right)$ then realizes the invariants. The problem of solving the cohomological equation (2.4) can be easily solved in the $\mathcal{C}^{\infty}$-smooth category by some sectorial germs $\varphi_{\alpha}$ exponentially tangent to identity (cf. $[1, \S 4.3]$ ). One then can obtain the bounded analytic solutions $\phi_{\alpha}(x)$ after correcting $\varphi_{\alpha}(x)$ by a $\mathcal{C}^{\infty}$ germ $g(x), \phi_{\alpha}=\varphi_{\alpha} \circ g^{\circ-1}$, obtained by the Ahlfors-Bers theorem as a solution to the Beltrami equation $\frac{\partial_{\bar{x}} g}{\partial_{x} g}=h$ on a neighborhood of the origin, where $h(x)$ is an exponentially flat $\mathcal{C}^{\infty}$ germ defined by $h:=\frac{\partial}{\partial \bar{x}} \varphi_{\alpha} / \frac{\partial}{\partial x} \varphi_{\alpha}$ which is independent of the sector $\Omega_{\alpha}$ since

$$
\begin{aligned}
0=\frac{\partial}{\partial \bar{x}} \widetilde{\psi}_{\beta}=\frac{\partial}{\partial \bar{x}}( & \left.\varphi_{\beta-\frac{\pi}{2 \nu}} \circ \varphi_{\beta+\frac{\pi}{2 \nu}}^{\circ-1}\right) \\
& =\left(\frac{\partial}{\partial \bar{x}} \varphi_{\beta-\frac{\pi}{2 \nu}}-\frac{\partial}{\partial x} \varphi_{\beta-\frac{\pi}{2 \nu}} \cdot \frac{\frac{\partial}{\partial \bar{x}} \varphi_{\beta+\frac{\pi}{2 \nu}}}{\frac{\partial}{\partial x} \varphi_{\beta+\frac{\pi}{2 \nu}}}\right) \cdot \frac{\partial}{\partial \bar{x}}\left(\bar{\varphi}_{\beta+\frac{\pi}{2 \nu}}^{-1}\right)
\end{aligned}
$$

by the chain rule for the Wirtinger derivative.

Remark. - In the formal equivalence problem for non-resonant irregular singularities one can assume that $\widehat{T}_{11}(0)=\widehat{T}_{12}(0)=1$, hence that $\widehat{g}(0)=0$, i.e. $\widehat{\phi}(x)=x+O\left(x^{\nu+2}\right)$. On the other hand, in the analytic equivalence problem one may have $\frac{\hat{T}_{12}(0)}{\hat{T}_{11}(0)} \neq 1$ and $\widehat{g}(0) \neq 0$, i.e. $\widehat{\phi}(x)=x+O\left(x^{\nu+1}\right)$. To be able to solve the equation for $\widehat{t}(x)$, one needs that $\widehat{\phi}(x)=x+O\left(x^{\nu+1}\right)$. This observation is at the heart of the following two examples.

Example 2.1. - (Formally equivalent but analytically non-equivalent resonant irregular singularities with analytically equivalent companion systems.) 
Consider the reducible equation of Poincaré rank $\nu=2$

$$
\left(\delta_{2}-\alpha_{2}(x)\right)\left(\delta_{2}-\alpha_{1}(x)\right) y=0,
$$

with

$$
\alpha_{1}(x)=1, \quad \alpha_{2}(x)=1+x+x^{2}+\frac{c x^{3}}{1+c x}, \quad c \in \mathbb{C} .
$$

Its basis of solutions is

$$
\begin{aligned}
& y_{1}(x)=e^{-\frac{1}{2 x^{2}}}, \quad x \in \mathbb{C}, \\
& y_{2}(x)=e^{-\frac{1}{2 x^{2}}} \int_{0+}^{x} e^{-\frac{1}{t}} \frac{1+c t}{t^{2}} \mathrm{~d} t=e^{-\frac{1}{2 x^{2}}} \int_{\frac{1}{x}}^{+\infty} e^{-s}\left(1+\frac{c}{s}\right) \mathrm{d} s, \quad x \in \mathbb{C} \backslash \mathbb{R}_{\leqslant 0},
\end{aligned}
$$

where the integration path in the $s$-variable follows horizontal rays. The projective Stokes matrices of the associated companion systems are easily calculated using the residue to be $S t_{0}=$ id and $S t_{\pi}=\left(\begin{array}{cc}1 & 2 \pi i c \\ 0 & 1\end{array}\right)$, which are conjugated by diagonal matrices for all $c \neq 0$. Up to analytic gauge transformation, the companion systems can be written as

$$
\delta_{2} v=\left(\begin{array}{cc}
\alpha_{1}(x) & 1 \\
0 & \alpha_{2}(x)
\end{array}\right) v,
$$

and are all formally equivalent to each other for all $c \in \mathbb{C}$ by a gauge transformation. Indeed, writing the formal gauge transformation between two such systems with $c$ and $\widetilde{c}$ as $v=\left(\begin{array}{ll}1 & \hat{f}(x) \\ 0 & \frac{1+c x}{1+\tilde{c} x}\end{array}\right) \widetilde{v}$, then $\widehat{f}(x)$ must satisfy

$$
\delta_{1} \widehat{f}=\frac{c-\widetilde{c}}{1+\widetilde{c} x}-\left(1+x+\frac{\widetilde{c} x^{2}}{1+\widetilde{c} x}\right) \widehat{f},
$$

which has a unique formal solution with $\widehat{f}(0)=c-\widetilde{c}$. Hence the companion systems are all analytically equivalent for $c, \widetilde{c} \neq 0$.

Also the equations are formally equivalent for all $c \in \mathbb{C}$ : indeed, it suffices to solve the conjugation equations

$$
e^{-\frac{1}{x}} \frac{1+c x}{x^{2}}=e^{-\frac{1}{\phi}} \frac{1+\widetilde{c} \phi}{\phi^{2}} \cdot \frac{\mathrm{d} \phi}{\mathrm{d} x}, \quad e^{-\frac{1}{2 x^{2}}}=\widehat{t}(x) e^{-\frac{1}{2 \phi^{2}}} .
$$

Writing $\phi(x)=x+x^{2} g(x)$, then $g(x)$ satisfies an analytic ODE

$$
\delta_{1} g=e^{-\frac{g}{1+x g}}(1+x g)^{2} \frac{1+c x}{1+\widetilde{c} x(1+x g)}-1-2 x g,
$$

with a "saddle-node" type singularity at $(x, g)=(0,0)$, which is known to have a unique formal solution $\widehat{g}(x)=(c-\widetilde{c}) x+O\left(x^{2}\right)$. Then $\widehat{t}(x)=$ $-\frac{c-\tilde{c}}{2}+O(x)$ can also be formally solved.

On the other hand, in the problem of analytic equivalence one needs to equalize the bases $c y_{1}(x), y_{2}(x)$ and $\widetilde{c}_{1}(\widetilde{x}), \widetilde{y}_{2}(\widetilde{x})$, with respect to which the 
Stokes matrices agree, and the conjugation equations

$$
e^{-\frac{1}{x}} \frac{1+c x}{c x^{2}}=e^{-\frac{1}{\phi}} \frac{1+\widetilde{c} \phi}{\widetilde{c} \phi^{2}} \cdot \frac{\mathrm{d} \phi}{\mathrm{d} x}, \quad c e^{-\frac{1}{2 x^{2}}}=\widetilde{c} \widehat{t}(x) e^{-\frac{1}{2 \phi^{2}}},
$$

are solved on sectors by the same $\phi(x)=x+\log \frac{\tilde{c}}{c} x^{2}+O\left(x^{3}\right)$ which is analytic as in the proof of Theorem 1.6. But now the equation for $t(x)$ has no formal solution if $c \neq \widetilde{c}$, and hence no analytic one either.

\section{Proof of Proposition 1.7. -}

(1). - Indeed, for a reducible $\operatorname{LDE}(1.14)$ one has $\Delta(x)=r(x)^{2}-$ $2 \delta_{\nu} r(x)$ for $r(x)=\alpha_{2}(x)-\alpha_{1}(x)$, and moreover $y_{1}(x)=e^{\int \alpha_{1}(x) \delta_{\nu}^{-1}}$ is convergent solution. Vice-versa, if $\Delta(x)=r(x)^{2}-2 \delta_{\nu} r(x)$ for an analytic $r(x)$, then the equation factors as (1.14) with $\alpha_{2}(x)=\frac{1}{2}(p(x)+r(x))$ and $\alpha_{1}(x)=\frac{1}{2}(p(x)-r(x))$. If $y(x)$ is a "convergent solution", then the equation factors as (1.14) with $\alpha_{1}(x)=\delta_{\nu} \log y(x)$ and $\alpha_{2}(x)=p(x)-\alpha_{1}(x)$, i.e. $r(x)=p(x)-2 \alpha_{1}(x)$. Finally, it is known that the companion system is analytically reducible, i.e. analytically equivalent to one in a triangular form, if and only if the Stokes matrices of are either all upper triangular or all lower triangular (indeed the formal diagonalizing transformation for a triangular system is triangular and therefore the Stokes matrices will have the same triangular form, and vice versa, a solution to the sectorial cohomological equation with triangular Stokes matrices exists that is triangular). The system $\delta_{\nu} v=A(x) v, A(x)=\left(a_{i j}(x)\right)$, realizing triangular Stokes data can be assumed upper triangular and with $a_{12}(0)=1$ (since $\left.a_{11}(0) \neq a_{22}(0)\right)$, and is therefore it is conjugated to $\delta_{\nu} v=\left(\begin{array}{cc}\alpha_{1}(x) & 1 \\ 0 & \alpha_{2}(x)\end{array}\right) v$ for some $\alpha_{1}(x), \alpha_{2}(x)$, hence analytically equivalent to the companion system of a reducible $\operatorname{LDE}(1.14)$.

(2). - The normal form LDE (1.20) has two linearly independent solutions $y_{j}(x)=e^{\left.\int \lambda_{j}(x) \delta_{\nu}^{(}-1\right)}, j=1,2$, and the equation (1.23) has an analytic solution $h(x)=\frac{1}{\lambda_{2}(x)-\lambda_{1}(x)}$. In general, if $y_{j}(x)=t_{j}(x) e^{\int \lambda_{j}(x) \delta_{\nu}^{-1}}, j=1,2$, is a pair of linearly independent solutions of the LDE, $f(x)=\frac{y_{2}(x)}{y_{1}(x)}$, then $h(x):=\frac{f}{\delta_{\nu} f}=\frac{1}{\lambda_{2}(x)-\lambda_{1}(x)+\delta_{\nu} \log \frac{t_{2}}{t_{1}}}$ is an analytic solution to (1.23).

Supposing that $h(x)$ is a nontrivial analytic solution to (1.23), let us show that the LDE is reducible. First, let us notice that from (1.23) it follows that $h(x)^{2} \Delta(x)=c^{2}+O\left(x^{2 \nu+1}\right)$ for some $0 \neq c \in \mathbb{C}$. Let $r(x, c):=\frac{c-\delta_{\nu} h(x)}{h(x)}$ and $\Delta\left(x, c^{2}\right):=r(x, c)^{2}-2 \delta_{\nu} r(x, c)=\frac{c^{2}-\left(\delta_{\nu} h(x)\right)^{2}+2 h(x) \delta_{\nu}^{2} h(x)}{h(x)^{2}}, c \in \mathbb{C}$. Considering the equation (1.23) as a non-homogeneous first order linear differential equation for unknown $\Delta$ with coefficients determined by $h(x)$, then $\Delta\left(x, a^{2}\right)$ are its solutions for all $a \in \mathbb{C}$ and for the reason of dimension there are 
no other solutions. Hence $\Delta(x)=\Delta\left(x, c^{2}\right)$ and the LDE is reducible, with $r(x)=r(x, \pm c)$, two different solution to $\Delta(x)=r(x)^{2}-2 \delta_{\nu} r(x)(1.22)$, and $y_{1}(x)=e^{\int \alpha_{1}(x) \delta_{\nu}^{-1}}, y_{2}(x)=h(x) e^{\int \alpha_{2}(x) \delta_{\nu}^{-1}}$, where $\alpha_{1}(x)=\frac{1}{2}(p(x)-r(x))$ and $\alpha_{2}(x)=\frac{1}{2}(p(x)+r(x))$ are two linearly independent convergent solutions to the LDE.

Vice-versa, if $r_{j}(x), j=1,2$, are two different solution to (1.22), then $h(x)=\frac{1}{r_{2}(x)-r_{1}(x)}, \delta_{\nu} \log h(x)=-\frac{r_{1}(x)+r_{2}(x)}{2}$, is an analytic solution to $(1.23)$.

Finally, a system with an irreducible irregular singularity and formal invariants $\left\{\lambda_{1}(x) \delta_{\nu}^{-1}, \lambda_{2}(x) \delta_{\nu}^{-1}\right\}$ is analytically equivalent to $\delta_{\nu} v=$ $\left(\begin{array}{cc}\lambda_{1}(x) & 0 \\ 0 & \lambda_{2}(x)\end{array}\right) v$, if and only if its collection of Stokes matrices is trivial, and one concludes by Theorem 1.6.

Proof of Theorem 1.8. - Two $2 \times 2$ linear differential systems with nonresonant irregular singularity at the origin of Poincaré rank $\nu=1$ are analytically equivalent if and only if they have the same formal invariants and the same trace of monodromy. Indeed, the monodromy matrix of such system with respect to the sectorial fundamental solution $V_{-\frac{\pi}{2}}(x)$ is of the form $M=\left(\begin{array}{cc}e^{2 \pi i \lambda_{1}^{(1)}} & 0 \\ 0 & e^{2 \pi i \lambda_{2}^{(1)}}\end{array}\right)\left(\begin{array}{cc}1 & 0 \\ s_{\pi} & 1\end{array}\right)\left(\begin{array}{cc}1 & s_{0} \\ 0 & 1\end{array}\right)$, where the product $s_{0} s_{\pi} \neq 0$ is the invariant characterizing the pair of Stokes matrices. Therefore $\operatorname{det} M=$ $e^{2 \pi i\left(\lambda_{1}^{(1)}+\lambda_{2}^{(1)}\right)}=e^{2 \pi i p^{(1)}}$ and the quantity

$(\operatorname{det} M)^{-\frac{1}{2}} \operatorname{tr} M=e^{\pi i\left(\lambda_{2}^{(1)}-\lambda_{1}^{(1)}\right.}\left(1+s_{0} s_{\pi}\right)+e^{\pi i\left(\lambda_{1}^{(1)}-\lambda_{2}^{(1)}\right.}=2 \cos \pi \mu+e^{\pi i \mu} s_{0} s_{\pi}$

is a natural invariant. On the other hand the eigenvalues of the residue matrix of the companion system at $x=\infty$ are $-\frac{p^{(1)}-1}{2} \pm \frac{1}{2} \sqrt{\Delta^{(2)}+1}$, where $\Delta^{(2)}=\left(p^{(1)}\right)^{2}+4 q^{(2)}-2 p^{(1)}$, and therefore

$$
(\operatorname{det} M)^{-\frac{1}{2}} \operatorname{tr} M=2 \cos \pi \sqrt{\Delta^{(2)}+1},
$$

from which one has (cf. [18, p. 144])

$$
s_{0} s_{\pi}=4 e^{-\pi i \mu} \sin \pi\left(\frac{\mu+\sqrt{\Delta^{(2)}+1}}{2}\right) \sin \pi\left(\frac{\mu-\sqrt{\Delta^{(2)}+1}}{2}\right) .
$$

For given formal and analytic invariants, the equation for $\Delta^{(2)}$ can be always solved.

If the LDE is reducible, written as (1.14), then there are only two analytic equivalence classes within a given formal equivalence class: one corresponds to both Stokes matrices trivial, and the other to one non-trivial Stokes matrix 
conjugated to $\left(\begin{array}{ll}1 & 1 \\ 0 & 1\end{array}\right)$. he equation has two canonical solutions:

$$
\begin{aligned}
& y_{1}(x)=e^{\int \alpha_{1}(x) \delta_{1}^{-1}}, \\
& y_{2}(x)=e^{\int \alpha_{1}(x) \delta_{1}^{-1}} \int_{0}^{x} e^{\int \alpha_{2}(x)-\alpha_{1}(x) \delta_{1}^{-1}} \delta_{1}^{-1},
\end{aligned}
$$

$x \in \mathbb{C} \backslash\left(\alpha_{2}^{(0)}-\alpha_{1}^{(0)}\right) \mathbb{R}_{\leqslant 0}$, where the integration path follows a real trajectory of $\frac{\delta_{1}}{\alpha_{2}(x)-\alpha_{1}(x)}$. Assuming that $\alpha_{2}(x)-\alpha_{1}(x)=1+\mu x+x^{2} r(x)$ for some analytic germ $r(x)$, then

$$
y_{2}(x)=y_{1}(x) \int_{0+}^{x} e^{-\frac{1}{x}} x^{\mu-2} R(x) \mathrm{d} x=y_{1}(x) \int_{\frac{1}{x}}^{+\infty} e^{-s} s^{-\mu} R\left(\frac{1}{s}\right) \mathrm{d} s,
$$

where $R(x):=e^{\int r(x) \mathrm{d} x}$, the integration in the second integral following a horizontal ray. Denoting $y_{2,+}(x)$, resp. $y_{2,-}(x)$, the branch of $y_{2}(x)$ on arg $x \in$ ]$\pi, 2 \pi[$, resp. $\arg x \in] 0, \pi[$, then for $\arg x=\pi$

$$
y_{2,+}(x)-y_{2,-}(x)=y_{1}(x) s_{\pi}, \quad s_{\pi}=\left(e^{2 \pi i \mu}-1\right) \int_{0}^{+\infty} e^{-s} s^{-\mu} R\left(\frac{1}{s}\right) \mathrm{d} s .
$$

In particular, if $\alpha_{2}(x)-\alpha_{1}(x)=1+\mu x$, i.e. $R(x)=1$, then $s_{\pi}=\left(e^{2 \pi i \mu}-\right.$ 1) $\Gamma(1-\mu)=e^{\pi i \mu} \frac{2 \pi i}{\Gamma(\mu)}$ which vanishes if and only if $\mu \in \mathbb{Z}_{\leqslant 0}$. And if $\alpha_{2}(x)-$ $\alpha_{1}(x)=1+\mu x-x^{2}$, i.e. $R(x)=e^{-x}$, then $s_{\pi}=\left(e^{2 \pi i \mu}-1\right) \sum_{j \geqslant 0}(-1)^{j} \Gamma(1-$ $\mu-j)=2 \pi i e^{\pi i \mu} \sum_{j \geqslant 0} \frac{1}{\Gamma(\mu+j)}$ which is positive for every $\mu \in \mathbb{Z}$.

\section{Non-degenerate resonant irregular singularities}

By Theorem 1.2, we may suppose $J_{0}^{\nu} \Delta(x)=x$ and let $J_{0}^{\nu} p(x)=: P(x)$, $P(0)=0$. The LDE may be rewritten as

$$
\delta_{\nu-\frac{1}{2}}^{2} y-\left(x^{-\frac{1}{2}} p(x)-\frac{1}{2} x^{\nu-\frac{1}{2}}\right) \delta_{\nu-\frac{1}{2}} y-x^{-1} q(x) y=0,
$$

which is non-resonant in the variable $x^{\frac{1}{2}}$. Correspondingly it has a formal solution basis

$$
\begin{aligned}
& \widehat{y}_{1}(x)=\widehat{T}_{11}\left(x^{\frac{1}{2}}\right) x^{-\frac{1}{4}} e^{\int \frac{1}{2}\left(P(x)-x^{\frac{1}{2}}\right) \delta_{\nu}^{-1}}, \\
& \widehat{y}_{2}(x)=\widehat{T}_{12}\left(x^{\frac{1}{2}}\right) x^{-\frac{1}{4}} e^{\int \frac{1}{2}\left(P(x)+x^{\frac{1}{2}}\right) \delta_{\nu}^{-1}},
\end{aligned}
$$

where $\widehat{T}_{11}(0)=\widehat{T}_{12}(0)=1$. By the results of the previous section, for every two such LDE for $y$ and $\widetilde{y}$ respectively there exists a unique formal transformation in $x^{\frac{1}{2}}$

$$
\widetilde{x}^{\frac{1}{2}}=\widehat{\phi}^{\frac{1}{2}}\left(x^{\frac{1}{2}}\right)=x^{\frac{1}{2}}+O\left(x^{\nu+\frac{1}{2}}\right), \quad \widetilde{y}=\widehat{t}\left(x^{\frac{1}{2}}\right) y, \widehat{t}(0)=1,
$$


that transforms the respective solutions (2.7) one to the other, and is Borel $(2 \nu-1)$-summable in the variable $x^{\frac{1}{2}}$.

Proof of Proposition 1.9. - Let us show that there exists a formal transformation in $x$

$$
\widetilde{x}=\widehat{\phi}(x)=x+O\left(x^{\nu+1}\right), \quad \widetilde{y}=\widehat{t}(x) y, \widehat{t}(0)=1,
$$

between the LDEs. This transformation will necessarily also transform the respective solutions (2.7) one to the other, so by the unicity it will agree with the above one. We can assume $\widetilde{\Delta}(\widetilde{x})=\widetilde{x}, \Delta(x)=x+O\left(x^{\nu+1}\right)$, and we construct the transformation $\widetilde{x}=\widehat{\phi}(x)$ as a formal infinite composition $\widehat{\phi}=\ldots \phi_{\nu+2} \circ \phi_{\nu+1} \circ \phi_{\nu}$, where $\phi_{k}(x)=x+a_{k} x^{k+1}$ is such that if $\Delta_{k}=x+$ $b_{k} x^{k+1}+O\left(x^{k+2}\right)$ and $\widetilde{x}=\phi_{k}(x)$ then $\Delta_{k+1}(\widetilde{x})=\widetilde{x}+O\left(\widetilde{x}^{k+2}\right)$. Plugging this into (1.11) gives $x+a_{k} x^{k+1}+O\left(x^{k}+2\right)=x+(2 k-2 \nu+1) b_{k} x^{k+1}+O\left(x^{k}+2\right)$, hence $b_{k}=\frac{a_{k}}{2 k-2 \nu+1}$ is uniquely determined. If $\widehat{\phi}(x)=x+O\left(x^{\nu+1}\right)$ and $p(x)=\tilde{p}(x)+O\left(x^{\nu+1}\right)$, then also the equation (1.9) for $\log \widehat{t}(x)$ has a unique formal solution.

Proof of Theorem 1.10. - If the Stokes operators agree, then by the reasoning of the proof of Theorem 1.6, the sectorial transformation between the respective solutions (2.7) of the two LDEs glue up to an analytic transformation in the variable $x^{\frac{1}{2}}$

$$
\widetilde{x}^{\frac{1}{2}}=\phi^{\frac{1}{2}}\left(x^{\frac{1}{2}}\right)=x^{\frac{1}{2}}+O\left(x^{\nu+\frac{1}{2}}\right), \quad \widetilde{y}=t\left(x^{\frac{1}{2}}\right) y, \widehat{t}(0)=1 .
$$

The Taylor expansion of this transformation is a formal transformation that is tangent to identity, hence by Proposition 1.9 it contains only whole powers of $x$, and therefore $\phi\left(x^{\frac{1}{2}}\right)$ and $t\left(x^{\frac{1}{2}}\right)$ are analytic also in $x$.

Proof of Theorem 1.11. - By the same reasoning as in the proof of Theorem 1.8. If $\nu=1$, then the equation (2.6) is of Poincaré rank 1 in the variable $x^{\frac{1}{2}}$.

Proof of Theorem 1.12. - Up to a composition with transformation $y \mapsto x^{m} y$, which changes $J_{0}^{\nu} p(x) \cdot \delta_{\nu}^{-1}$ by $m \delta_{0}^{-1}$ while preserving the monodromy and the Stokes operators, the meromorphic classification problem is reduced to the analytic one. In fact, in the irregular case meromorphic transformations preserve the canonical sectorial solution bases.

Proof of Theorem 1.13. - Calculating the coefficients of $\partial_{x}$ and $\partial_{y}$ in (1.29) with (1.30) and (1.31) both give $\alpha=\frac{\mathrm{d}}{\mathrm{d} x} g$. Then the coefficients 
of $y \partial_{y_{x}}$ and $y_{x} \partial_{y_{x}}$ in (1.29) give respectively

$$
\begin{aligned}
& 2 \frac{q}{x^{2 \nu+2}} \frac{\mathrm{d}}{\mathrm{d} x} g+g \frac{\mathrm{d}}{\mathrm{d} x}\left(\frac{q}{x^{2 \nu+2}}\right)=\frac{\mathrm{d}^{2}}{\mathrm{~d} x^{2}} f-\left(\frac{p}{x^{\nu+1}}-\frac{\nu+1}{x}\right) \frac{\mathrm{d}}{\mathrm{d} x} f \\
& \frac{\mathrm{d}^{2}}{\mathrm{~d} x^{2}} g+\left(\frac{p}{x^{\nu+1}}-\frac{\nu+1}{x}\right) \frac{\mathrm{d}}{\mathrm{d} x} g+g \frac{\mathrm{d}}{\mathrm{d} x}\left(\frac{p}{x^{\nu+1}}-\frac{\nu+1}{x}\right)=2 \frac{\mathrm{d}}{\mathrm{d} x} f .
\end{aligned}
$$

Hence

$$
2 f(x)=\frac{\mathrm{d}}{\mathrm{d} x} g+\left(\frac{p(x)}{x^{\nu+1}}-\frac{\nu+1}{x}\right) g(x)+2 f^{(0)}, \quad \text { for some } f^{(0)} \in \mathbb{C} .
$$

Let $h(x)=\frac{g(x)}{x^{\nu+1}}$, then $2 f=\delta_{\nu} h+p h+2 f^{(0)}$, and $\delta_{\nu}^{2} f-p \delta_{\nu} f=2 q \delta_{\nu} h+\delta_{\nu} q h$, from which

$$
\delta_{\nu}^{3} h-\Delta \delta_{\nu} h-\frac{1}{2} \delta_{\nu} \Delta h=0 .
$$

For a strictly non-resonant regular singularity (1.15), the equation to solve is

$$
\delta_{0}^{3} h-\left(\lambda_{1}-\lambda_{2}\right)^{2} \delta_{0} h=0,
$$

which has a basis of solutions $h_{1}(x)=1, h_{2}(x)=x^{\lambda_{1}-\lambda_{2}}, h_{3}(x)=x^{\lambda_{2}-\lambda_{1}}$ if $\lambda_{1} \neq \lambda_{2}$, and $h_{1}(x)=1, h_{2}(x)=\log x, h_{3}(x)=(\log x)^{2}$ if $\lambda_{1}=\lambda_{2}$.

For a resonant regular singularity (1.17), the equation for $h$ is of the form (1.24) with $\nu=0$ and $r(x)=-\frac{k}{1-x^{k}}$, which has a basis of solutions $h_{1}(x)=\frac{x^{k}}{1-x^{k}}, h_{2}(x)=\frac{1}{1-x^{k}}+\frac{k x^{k}}{1-x^{k}} \log x$, and $h_{3}(x)$ given by $\left(\delta_{0}+\right.$ $r(x)) h_{3}(x)=\frac{1}{x^{k}}+k \log x$.

If $\nu>0$ and $\Delta(0) \neq 0$, then by Proposition 1.7 the equation (1.23) has a non-trivial analytic solution $h(x)$ if and only if the LDE is analytically equivalent to its formal normal form (1.20), for which one has $h(x)=\frac{c}{\lambda_{2}(x)-\lambda_{1}(x)}$, $c \in \mathbb{C}$.

\section{Appendix: Implicit function theorem for Borel summable power series}

We will recall the notion of Borel summability and present a Borel summable version of the implicit function theorem.

There are several equivalent ways to define Borel summability (see e.g. [2, $13,17,18,22])$. We will use the following one due to J.-P. Ramis.

Let $(E,\|\cdot\|)$ be a Banach space: we will consider the following two, (i) the field $(\mathbb{C},|\cdot|)$, (ii) the space of bounded analytic functions on some small disc $D=\{|y| \leqslant \epsilon\}, \epsilon>0$, together with supremum norm. 
Let $\widehat{f}(x)=\sum_{n \geqslant 0} f^{(n)} x^{n} \in E \llbracket x \rrbracket$ be a formal power series with coefficients $f^{(n)} \in E$.

- An open sectorial domain at the origin is a simply connected domain $U$ in $\mathbb{C}$ (or in the Riemann surface of logarithm) with 0 in its boundary that can be written as an (infinite) union of open sectors at 0 of increasing angular opening (and decreasing radius).

- Let $U$ be an open sectorial domain at the origin. An analytic function $f: U \rightarrow E$ is said to be $s$-Gevrey asymptotic to $\widehat{f}, s>0$, if for every sector $V \subset \subset U$ (i.e. such that $\bar{V} \subset U \cup\{0\}$ ) there exist $C, A>0$ such that

$\left\|f(x)-\sum_{n=0}^{N-1} f^{(n)} x^{n}\right\| \leqslant C|x|^{N} A^{N} \Gamma(1+s N), \quad$ for all $N>0$ and all $x \in V$.

- An analytic function $f: U \rightarrow E$ is said to be exponentially flat of order $\nu>0$ if it is $\frac{1}{\nu}$-Gevrey asymptotic to the zero series. This is equivalent to ask that for every sector $V \subset \subset U$ there exist $C, A>0$ such that

$$
\|f(x)\| \leqslant C e^{-\frac{A}{|x|^{\nu}}}, \quad \text { for all } x \in V .
$$

- The formal power series $\widehat{f}(x)$ is said to be Borel $\nu$-summable in a direction $\alpha \in \mathbb{R}$ for $\nu>0$, if there exists an open sector $U$ of angular width $>\frac{\pi}{\nu}$ bisected by $\alpha$, and a function $f_{\alpha}: U \rightarrow E$ that is $\frac{1}{\nu}$-Gevrey asymptotic to $\widehat{f}(x)$. It is said to be Borel $\nu$-summable if it is summable in all directions $\alpha \in[0,2 \pi[$ up to finitely many. The directions of non-summability are called anti-Stokes or singular.

Let $\beta_{0}<\ldots<\beta_{m-1}$ be the anti-Stokes directions of a Borel $\nu$-summable $\widehat{f}(x)$ in the interval $\left[0,2 \pi\left[, \beta_{m}=\beta_{0}+2 \pi\right.\right.$. For $\eta>0$ arbitrarily small, let

$$
\begin{aligned}
V_{j}=\{\arg x \in] & \beta_{j}-\frac{\pi}{2 \nu}+\eta, \beta_{j+1}+\frac{\pi}{2 \nu}-\eta[,|x|<\rho(\eta)\}, \\
& j=0, \ldots, m-1,
\end{aligned}
$$

with some $\rho(\eta)>0$, be a cyclic covering of a pointed neighborhood of 0 by sectors, and $f_{V_{j}}: V_{j} \rightarrow E$ the Borel sum of $\widehat{f}$ in the directions $\alpha \in$ ]$\beta_{j}+\eta, \beta_{j+1}-\eta$. Then by definition $f_{V_{j+1}}-f_{V_{j}}$ is exponentially flat of order $\nu$ on the intersection $V_{j} \cap V_{j+1}$.

By the Ramis-Sibuya theorem the converse is also true, giving thus a useful characterization of Borel summability.

TheOREM (Ramis-Sibuya). - Let $V_{j}, j \in \mathbb{Z}_{m}$, be a cyclic covering of a pointed neighborhood of 0 by sectors. Let $f_{V_{j}, V_{j+1}}: V_{j} \cap V_{j+1} \rightarrow E$ be 
exponentially flat of order $\nu$, for all $j \in \mathbb{Z}_{m}$. Then there exists a formal power series $\widehat{f}(x)$, and a "cochain" of sectorial functions $f_{V_{j}}: V_{j} \rightarrow E, j \in \mathbb{Z}_{m}$, that are $\frac{1}{\nu}$-Gevrey asymptotic to $\widehat{f}(x)$, such that $f_{V_{j}, V_{j+1}}=f_{V_{j+1}}-f_{V_{j}}$.

In particular, if the angular opening of $V_{j}$ is $>\frac{\pi}{\nu}$, then $\widehat{f}(x)$ is Borel $\nu$-summable in the directions covered by $e^{-\frac{\pi}{2 \nu}} V_{j} \cap e^{\frac{\pi}{2 \nu}} V_{j}$.

Proposition 2.2 (Implicit function theorem). - Let $\widehat{F}(x, y)=$ $\sum_{j \geqslant 0} F^{(j)}(y) x^{j}$ be a formal power series of $x$ such that the coefficients $F^{(j)}(y)$ bounded and analytic in $y$ on some small disc $D=\{|y| \leqslant \epsilon\}$, $\epsilon>0$, and the series is Borel $\nu$-summable. Suppose that $F^{(0)}(0)=0$ and $\frac{\mathrm{d} F^{(0)}}{\mathrm{d} y}(0) \neq 0$. Then the implicit equation

$$
\widehat{F}(x, g(x))=0,
$$

has a unique formal solution $\widehat{g}(x)=\sum_{j \geqslant 0} g^{(j)} x^{j}, g^{(0)}=0$, which is Borel $\nu$ summable with singular directions among the singular directions of $\widehat{F}(x, y)$.

The above proposition follows directly from a sectoral implicit function theorem with Gevrey asymptotics that is stated in [19, Theorem 2.8] with a reference to the book [14], and also proved in [6]. Some related statements can be also found in [26]. We provide a sketch of a proof below.

Proof. - For any small $\eta>0$, let $F_{V_{j}}: V_{j} \times D \rightarrow \mathbb{C}, j \in \mathbb{Z}_{m}$, be the "cochain" of the Borel sums of $\widehat{F}$ on the cyclic sectorial covering $V_{j}(2.8)$. Then there exist $C, A>0$ such that

$$
\begin{aligned}
\left|F_{V_{j+1}}(x, y)-F_{V_{j}}(x, y)\right| \leqslant & C e^{-\frac{A}{|x|^{D}}} \\
& \text { on the intersections }(x, y) \in\left(V_{j} \cap V_{j+1}\right) \times D .
\end{aligned}
$$

We shall solve the sectorial implicit equations

$$
F_{V_{j}}\left(x, g_{V_{j}}(x)\right)=0,
$$

for $x \in V_{j}$, by replicating the usual proof of the implicit function theorem. The sectorial solution $g_{V_{j}}(x)$ is obtained as a fixed point of the operator $\mathcal{K}_{j}: y(x) \mapsto y(x)-\left(\frac{\partial F^{(0)}}{\partial y}(0)\right)^{-1} F_{V_{j}}(x, y(x)), \quad g_{V_{j}}(x)=\lim _{n \rightarrow+\infty} \mathcal{K}_{j}^{\circ n}(x, 0)$,

on the space of bounded analytic functions on the sector $V_{j}$ with $\sup _{x \in V_{j}}|y(x)| \leqslant \frac{\epsilon}{2}$ for some small $\epsilon>0$, supposing that the radius of $V_{j}$ 
is small enough. Indeed, let us estimate

$$
\begin{aligned}
\left|\frac{\partial \mathcal{K}_{j}}{\partial y}(x, y)\right| \leqslant\left|\frac{\partial F^{(0)}}{\partial y}(0)\right|^{-1} \mid \frac{\partial F_{V_{j}}}{\partial y}(x, y) & -\frac{\partial F^{(0)}}{\partial y}(y) \mid \\
& +\left|\left(\frac{\partial F^{(0)}}{\partial y}(0)\right)^{-1} \frac{\partial F^{(0)}}{\partial y}(y)-1\right|,
\end{aligned}
$$

for $x \in V_{j},|y|<\frac{\epsilon}{2}$. The second term can be made arbitrarily small by restricting the radius $\epsilon$ of $D$. The first term can expressed by the Cauchy formula as

$$
\frac{1}{2 \pi}\left|\frac{\partial F^{(0)}}{\partial y}(0)\right|^{-1}\left|\int_{|\zeta-y|=\frac{\epsilon}{2}} \frac{F_{V_{j}}(x, \zeta)-F^{(0)}(\zeta)}{\zeta-y} \mathrm{~d} \zeta\right| \leqslant c|x|,
$$

for some $c>0$. Hence, up to restricting the radius $\rho(\eta)$ of the sector $V_{j}$, one can assume that $\left|\frac{\partial \mathcal{K}_{j}}{\partial y}(x, y)\right| \leqslant \frac{1}{2}$ for $x \in V_{j},|y|<\frac{\epsilon}{2}$, and the operator is contractive.

Let us now show that $\left|g_{V_{j+1}}(x)-g_{V_{j}}(x)\right| \leqslant K e^{-\frac{A}{|x|^{\nu}}}$ on the intersections $V_{j+1} \cap V_{j}$ for some $K>0$ in order to apply the Ramis-Sibuya theorem and obtain the Borel summability. For $x \in V_{j+1} \cap V_{j}$ and $\left|y_{j+1}\right|,\left|y_{j}\right| \leqslant \frac{\epsilon}{2}$ one can estimate $\left|\mathcal{K}_{j+1}\left(x, y_{j+1}\right)-\mathcal{K}_{j}\left(x, y_{j}\right)\right| \leqslant\left|\mathcal{K}_{j+1}\left(x, y_{j+1}\right)-\mathcal{K}_{j+1}\left(x, y_{j}\right)\right|+$ $\left|\mathcal{K}_{j+1}\left(x, y_{j}\right)-\mathcal{K}_{j}\left(x, y_{j}\right)\right|$, where the first term is bounded by $\left|y_{j+1}-y_{j}\right| \cdot$ $\int_{0}^{1}\left|\frac{\partial \mathcal{K}_{j}}{\partial y}\left(x, t y_{j+1}+(1-t) y_{j}\right)\right| \mathrm{d} t \leqslant \frac{1}{2}\left|y_{j+1}-y_{j}\right|$, and the second term is bounded by $\left|\frac{\partial F^{(0)}}{\partial y}(0)\right|^{-1} C e^{-\frac{A}{|x|^{D}}}$. Therefore the difference of all the respective iterations satisfy $\left|\mathcal{K}_{j+1}^{\circ n}(x, 0)-\mathcal{K}_{j}^{\circ n}(x, 0)\right| \leqslant K e^{-\frac{A}{|x|^{\nu}}}$ for $K=2\left|\frac{\partial F^{(0)}}{\partial y}(0)\right|^{-1} C$ and so do the limits $\left|g_{V_{j+1}}(x)-g_{V_{j}}(x)\right| \leqslant K e^{-\frac{A}{|x|^{\nu}}}$.

\section{Acknowledgement}

The motivation to look at analytic classification of second order linear differential equations came from the article [10] of Kossovskiy \& Schafikov where the question of analytic point equivalence was posed and a particular case investigated in connection to a classification problem in CR geometry.

I am happy to acknowledge the support of Ilya Kossovskiy from his grant P294682 of The Austrian Science Fund (FWF) during the preparation of this paper, and would like to thank him for his encouragement and generosity. I also want to thank the Department of Mathematics at the Masaryk University in Brno and the Faculty of Mathematics at the University of Vienna for their hospitality. 


\section{Bibliography}

[1] D. G. Babbitt \& V. S. Varadarajan, Local moduli for meromorphic differential equations, Astérisque, vol. 169-170, Société Mathématique de France, 1989.

[2] W. BALSER, Formal power series and linear systems of meromorphic ordinary differential equations, Universitext, Springer, 2000.

[3] W. Balser, W. B. Jurkat \& D. A. Lutz, "A general theory of invariants for meromorphic differential equations. I: Formal invariants, II: Proper invariants", Funkc. Ekvacioj, Ser. Int. 22 (1979), p. 197-221 and 257-283.

[4] G. D. BiRkhoff, "The generalized Riemann problem for linear differential equations and the allied problems for linear difference and $q$-difference equations", Proc. Am. Acad. 49 (1913), p. 521-568.

[5] D. Blázquez-Sanz, J. J. Morales-Ruiz \& J.-A. Weil, "Differential Galois theory and Lie symmetries", SIGMA, Symmetry Integrability Geom. Methods Appl. 11 (2015), article no. 092 (17 pages).

[6] P. De Maesschalck, "Geometry and Gevrey asymptotics of two-dimensional turning points", PhD Thesis, Hasselt University (Belgium), 2003.

[7] L. E. Dickson, "Differential equations from the group standpoint", Ann. Math. 25 (1924), p. 287-378.

[8] N. S. Hawley \& M. Schiffer, "Half-order differentials on Riemann surfaces", Acta Math. 115 (1966), p. 199-236.

[9] Y. Ilyashenko \& S. Yakovenko, Lectures on analytic differential equations, Graduate Studies in Mathematics, vol. 86, American Mathematical Society, 2008.

[10] I. Kossovskiy \& R. Shafikov, "Divergent CR-equivalences and meromorphic differential equations", J. Eur. Math. Soc. 18 (2016), no. 12, p. 2785-2819.

[11] E. Kummer, "De generali quadam equatione differentiali tertii ordinis", J. Reine Angew. Math. 100 (1887), p. 1-9, first published in 1834.

[12] E. N. LAGUerRe, "Sur quelques invariants des équations différentielles linéaires", $C$. R. Acad. Sci. Paris 88 (1879), p. 424-427.

[13] M. Loday-Richaud, Divergent Series, Summability and Resurgence II: Simple and Multiple Summability, Lecture Notes in Mathematics, vol. 2154, Springer, 2016.

[14] H. Majima, Asymptotic analysis for integrable connections with irregular singular points, Lecture Notes in Mathematics, vol. 1075, Springer, 1984.

[15] B. Malgrange, "Remarques sur les équations différentielles à points singuliers irréguliers", in Équations différentielles et systèmes de Pfaff dans le champ complexe, Lecture Notes in Mathematics, vol. 712, Springer, 1979, p. 77-86.

[16] _ , "Travaux d'Ecalle et de Martinet-Ramis sur les systèmes dynamiques", in Séminaire Bourbaki, vol. 1981/82, Astérisque, vol. 92-93, Société Mathématique de France, 1982, p. 59-73.

[17] — "Sommation des séries divergentes", Expo. Math. 13 (1995), no. 2-3, p. 163222.

[18] J. Martinet \& J.-P. Ramis, "Problémes de modules pour des équations différentielles non linéaires du premier ordre", Publ. Math., Inst. Hautes Étud. Sci. 55 (1982), p. 63164 .

[19] J. Mozo-Fernández, "Weierstrass theorems in strong asymptotic analysis", Bull. Pol. Acad. Sci., Math. 49 (2001), no. 3, p. 255-268.

[20] W. R. Oudshoorn \& M. van DER Put, "Lie symmetries and differential galois groups of linear equations", Math. Comput. 71 (2002), no. 237, p. 349-361.

[21] M. van DeR Put \& M. F. Singer, Galois Theory of Linear Differential Equations, Grundlehren der Mathematischen Wissenschaften, vol. 328, Springer, 2003. 


\section{Martin Klimeš}

[22] Y. SiBUYA, Linear differential equations in the complex domain : problems of analytic continuation, Translations of Mathematical Monographs, vol. 82, American Mathematical Society, 1990.

[23] P. StÄCkel, "Ueber Transformationen von Differentialgleichungen", J. Reine Angew. Math. 111 (1893), p. 290-302.

[24] K. Strebel, Quadratic differentials, Ergebnisse der Mathematik und ihrer Grenzgebiete. 3. Folge., vol. 5, Springer, 1984.

[25] S. Tanny \& S. Yakovenko, "On local Weyl equivalence of higher order Fuchsian equations", Arnold Math. J. 1 (2015), no. 2, p. 141-170.

[26] J.-C. Tougeron, "Sur les ensembles semi-analytiques avec conditions Gevrey au bord", Ann. Sci. Éc. Norm. Supér. 27 (1994), no. 2, p. 173-208. 